\title{
Sensory Recruitment in Visual Short-Term Memory: A Systematic Review and Meta-Analysis of Sensory Visual Cortex Interference Using Transcranial Magnetic Stimulation
}

Phylactou, P. ${ }^{1}$, Traikapi, A. ${ }^{1}$, Papadatou-Pastou, $M .^{2,3} \&$ Konstantinou, $N .^{1}$

${ }^{1}$ Department of Rehabilitation Sciences, Faculty of Health Sciences, Cyprus University of Technology

${ }^{2}$ Department of Special Education and Psychology, National and Kapodistrian University of Athens, Athens, Greece

${ }^{3}$ Biomedical Research Foundation of the Academy of Athens, Athens, Greece

Draft version 6.1, 23.05.2022.

This preprint has not undergone any post-submission improvements or corrections. The

Version of Record of this article is published in Psychonomic Bulletin \& Review, and is available online at https://doi.org/10.3758/s13423-022-02107-y

Corresponding author: Phivos Phylactou, Department of Rehabilitation Sciences, Cyprus University of Technology, 15 Vragadinou Street, $5^{\text {th }}$ floor office 517, Limassol, 3041, Cyprus. Email: pp.phylactou@edu.cut.ac.cy 


\begin{abstract}
Sensory visual areas are involved in encoding information in Visual Short-Term Memory (VSTM). Yet, it remains unclear if sensory visual cortex is a necessary component of the brain network for maintenance of information in VSTM. Here, we aimed to systematically review studies that have investigated the role of the sensory visual cortex in VSTM using transcranial magnetic stimulation (TMS), and to quantitatively explore these effects using meta-analyses. Fourteen studies were identified and reviewed. Eight studies provided sufficient data for meta-analysis. Two meta-analyses, one regarding the VSTM encoding phase (17 effect sizes) and one regarding the VSTM maintenance phase (15 effect sizes), two meta-regressions (32 effect sizes in each), and one exploratory meta-analysis were conducted. Our results indicate that the sensory visual cortex is similarly involved in both the encoding and maintenance VSTM phase. We suggest that in some cases where evidence did not show significant TMS effects, this is due to low memory or perceptual task demands. Overall, these findings support the idea that sensory visual areas are part of the brain network responsible for successfully maintaining information in VSTM.
\end{abstract}

Keywords: primary visual cortex, early visual cortex, visual short-term memory, systematic review, meta-analysis, sensory recruitment. 
Is sensory visual cortex a necessary component of the network that underlies the short-term maintenance or storage of visual information? It is well-established that visual short-term memory (VSTM) is associated with frontal (Christophel et al., 2017; Funahashi, 2017; Levy, \& Goldman-Rakic, 2000; Riley, \& Constantinidis, 2016) and parietal (Bettencourt, \& Xu, 2016; Ester et al., 2015, 2016; Konstantinou et al., 2016; Xu, \& Chun, 2006) brain areas. However, the role of the sensory visual areas (e.g., early visual areas such as $\mathrm{V} 1, \mathrm{~V} 2$ or $\mathrm{V} 5 / \mathrm{MT}+$ ) in short-term maintenance of visual information is still unclear (e.g., Scimeca et al., 2018; Xu, 2017, 2020, 2021). Recent formulations of the debate focus on whether sensory visual areas are employed only during encoding and consolidation of visual information (Bays et al., 2011; Brady et al., 2016; Kammer, 2007; Vogel et al., 2006; Ye et al., 2017, 2020) or if they are also engaged during the short-term maintenance of such information (Konstantinou et al., 2012).

Central to this debate is the sensory recruitment hypothesis, according to which early visual areas are necessary for the successful maintenance of information in VSTM (Harrison, \& Tong, 2009; Serences et al., 2009; Supèr et al., 2001; for reviews see Lorenc, \& Sreenivasan, 2021; Pasternak, \& Greenlee, 2005; Postle, 2006, 2015, 2016; Serences, 2016; Teng, \& Postle, 2021). The sensory recruitment hypothesis is supported by evidence from primate and human studies (e.g., Awh \& Jonides, 2001; Christophel et al., 2017, 2018; Harrison, \& Tong, 2009; Lorenc et al., 2018; Pasternak, \& Greenlee, 2005; Postle, 2006; Rademaker et al., 2019; Serences, 2016; Serences et al., 2009; Sreenivasen et al., 2014; Supèr et al., 2001), suggesting that the sensory visual cortex is not only involved in the encoding of visual information, but also in the successful maintenance of it. Yet, recent studies have provided evidence that irrelevant visual distraction has minimal impact on VSTM (Bettencourt, \& Xu, 2016; for recent reviews see Xu, 2017, 2020, 2021) suggesting that the sensory visual cortex is not essential for the successful short-term maintenance of visual information (Ester et al., 2015, 2016; Lee et al., 2013; Mendoza-Halliday et al., 2014; Stokes, 2015; for a recent review see Riley, \& Constantinidis, 2016). Indeed, recent qualitative reviews of primate and human studies, suggested that the current evidence does not support the idea that the engagement of the sensory visual cortex in the maintenance of visual information is required, but higher order cortical areas (i.e., prefrontal cortex and posterior parietal cortex) are most likely responsible for the short-term maintenance of visual information (Xu, 2017, 2020, 2021).

The role of the sensory visual cortex during the encoding and consolidation of information in VSTM is well studied (Awh \& Jonides, 2001; D'Esposito, \& Postle, 2015; de 
Graaf et al., 2014; Kammer, 2007; Masse et al., 2020; Serences, 2016; Shevlin, 2020; Xu, 2017, 2020, 2021), and evidence from studies employing functional magnetic resonance imaging (fMRI; e.g., Bettencourt, \& Xu, 2016), electroencephalography (EEG; e.g., Tcheslavski et al., 2018), brain stimulation (e.g., Lee et al., 2016) together with non-human primate studies (e.g., Lu et al., 2018) have linked activity in the sensory visual cortex with successful encoding and consolidation of visual information in VSTM. Yet, the role of the sensory visual cortex in VSTM maintenance remains controversial.

Recently, Xu $(2017,2018,2020,2021)$ argued that the sensory visual cortex is unable to maintain VSTM representations for two main limitations. Firstly, Xu $(2017,2018,2020$, 2021) describes that given the essential role of the sensory visual cortex in encoding and consolidation of visual information (see Awh \& Jonides, 2001; D’Esposito \& Postle, 2015; de Graaf et al., 2014; Kammer, 2007; Masse et al., 2020; Serences, 2016; Shevlin, 2020), such information maintenance by the sensory visual cortex makes representations susceptible to overwriting as new stimuli are processed. Counterarguments which attempted to reaffirm the sensory recruitment hypothesis (e.g., Gayet et al., 2018; Scimeca et al., 2018), proposed that the sensory visual cortex utilizes processes to protect representations, such as between layer top-down signals in area V1 (van Kerkoerle et al., 2017; see also Zhao et al., 2021). These processes were described as similar to those employed by higher brain areas, such as the prefrontal cortex when differentiating mnemonic and perceptual information during attention modulation (e.g., Knight et al., 1999), proposing that similar mechanisms to segregate between perception and memory are utilized by the sensory visual cortex (see Scimeca et al., 2018). Moreover, it has been postulated that instead of impairing VSTM, the interaction between memory representations and perceptual input might instead be beneficial. For instance, VSTM representations can bias perceptual input, thus improving perceptual continuity and goal-related behavior (Gayet et al., 2013; Kiyonaga et al., 2017). Secondly, Xu (2017) pointed out that sensory visual cortex is not sufficiently wired to support the type of sustained activity thought to support VSTM. However, alternative explanations suggested that sustained activity in the prefrontal cortex might not in fact reflect VSTM representations per se, but instead echo a biasing signal to protect or direct attention towards goal related VSTM representations (Curtis \& D'Esposito, 2003; Sreenivasan \& D’Esposito, 2019; Masse et al., 2020; Miller \& Cohen, 2001; Sreenivasan et al., 2014).

Here, we suggest that the controversial role of the sensory visual cortex in VSTM maintenance is due to methodological differences between relevant studies, such as maintenance periods that vary considerably between VSTM experiments from a few hundred 
milliseconds up to a few seconds (for a review see, van de Ven \& Sack, 2013), and due to the fact that the neuroimaging methods employed for measuring such dynamic content-specific delay activity (i.e., fMRI and EEG) lack the precision to detect subtle or activity-silent processes (e.g., Rose et al., 2016; Stokes, 2015; Zhang et al, 2021; see also Oberauer, 2019; Serences, 2016; Sreenivasen et al., 2014; for a recent review see Masse et al., 2020). Recently, it has been proposed that further to the conventional sustained-activity storage view (see Leavitt et al., 2017), representations in VSTM can be protected via activity-silent processes, such as through changes in synaptic weights (Sreenivasan \& D'Esposito, 2019; Masse et al., 2020; Lorenc et al., 2021; Lorenc \& Sreenivasan, 2021; Teng \& Postle, 2021; see also Beukers et al., 2021). Even though activity-silent processes were initially introduced for frontal (Stokes, 2015) and parietal (Rose et al., 2016) areas, recent evidence points to activity-silent storage in sensory visual areas as well (Iamshchinina et al., 2021; Lorenc et al., 2018). These limitations, fail to exclude the possibility of sensory visual cortex involvement even in tasks that show little or no sustained activity using fMRI during the maintenance period, making it unclear if in addition to its well-established role in encoding, sensory visual cortex is also causally involved in the short-term maintenance of visual information.

Several previous attempts to reconcile disparate lines of evidence focused on qualitative reviews that lack a systematic approach of study identification, thus leaving any conclusions open to bias (Awh \& Jonides, 2001; Christophel et al., 2017; D'Esposito, \& Postle, 2015; Ester et al., 2016; Gayet et al., 2018; Lorenc, \& Sreenivasan, 2021; Scimeca et al., 2018; Serences, 2016; Sreenivasen et al., 2014; Tapia \& Beck, 2014; Teng, \& Postle, 2021; van de Ven \& Sack, 2013; Xu, 2017, 2018, 2020, 2021; see also Shevlin, 2020). Furthermore, most of these reviews relied heavily on neuroimaging data, which cannot provide causal information as to the question of whether the sensory visual cortex is indeed a necessary component of the network that underlies the successful short-term maintenance of visual information (Masse et al., 2020; Serences, 2016; Sreenivasen et al., 2014; Xu, 2017; see also D'Esposito et al., 1999).

In the current systematic review and meta-analyses we address these limitations by systematically identifying human studies that employed transcranial magnetic stimulation (TMS). An ideal scenario for causal evidence of the involvement of sensory visual cortex in the short-term maintenance of visual representations would be to completely inactivate the sensory visual cortex throughout the maintenance period of a VSTM task and reactivate it immediately before a memory probe is presented for comparison or matching (for a similar argument see Scimeca et al., 2018), but this scenario remains a hypothetical one since it is 
impossible to be carried out. However, using non-invasive brain stimulation, such as TMS, during the delay period of a VSTM task, it is possible to interfere with sensory visual cortex activity and assess the effects of such interference on the behavioral performance of the VSTM task. Any significant interference due to TMS over sensory visual cortex against baseline VSTM performance can be taken as causal evidence for the involvement of sensory visual cortex in the brain network responsible for short-term maintenance of visual information (Scimeca et al., 2018). TMS is a non-invasive method that uses a coil to deliver magnetic pulses that can interfere with neural activity in specific brain regions with good spatial and temporal resolution (e.g., the "virtual lesion" or "neural noise" methods; Harris et al., 2008; see also de Graaf, \& Sack, 2011; Hallett, 2000; Pascual-Leone et al., 2000; Pitcher et al., 2020; Sack, 2006; Sandrini et al., 2011; Siebner et al., 2009) and has been shown to either inhibit brain processing or enhance neural excitability (e.g., Kim et al., 2015; Moliadze et al., 2003; Silvanto et al., 2018; for reviews see Robertson et al., 2003; Silvanto \& Cattaneo, 2017). Thus, TMS can furnish causal information about the relationship between the brain network underlying behavioral responses, as opposed to the correlational nature of neuroimaging data (for recent reviews see Bergmann, \& Hartwigsen, 2021; Pitcher et al., 2020). This gap between the correlational nature of neuroimaging data and the causal link of TMS, has been demonstrated since the introduction of TMS in cognitive science (for a review see Robertson et al., 2003). For example, initial neuroimaging evidence has led to the conclusion that the involvement of the pre-frontal cortex during sequence learning was required only after awareness for the sequence had been achieved (Clegg et al., 1998). It was only after the introduction of TMS in this line of work that the role of the pre-frontal cortex was established during the absence of awareness for sequence learning (e.g., Robertson et al., 2001). Similarly, the role of feedforward and feedback processes in area V1, remained controversial (e.g., Lamme et al., 2000), until Pascual-Leone and Walsh (2001) successfully applied TMS to interfere with back-projections between area V5/MT+ and V1.

As discussed in detail below (see Systematic Review), previous TMS studies that aimed to explore the role of the sensory visual cortex during VSTM differentiated between the initial encoding-consolidation phases and the maintenance phase (e.g., Rademaker et al., 2017; van de Ven et al., 2012; van Lamsweerde et al., 2017; see also Xu, 2017). Specifically, the separation between encoding-consolidation and maintenance adopted in previous TMS studies is in line with evidence for a two-stage consolidation process in VSTM, which describes that, in order to store representations in VSTM, representations are initially allocated minimum resources in the early consolidation stage, but gradually receive more 
resources in a later consolidation stage if more encoding time is given (Ye et al., 2017, 2020). The second stage of information encoding and consolidation in VSTM might still take place for up to $200 \mathrm{~ms}$ after stimulus offset, due to memory load (e.g., Jolicoeur \& Dell'Acqua, 1998; Vogel et al., 2006) and/or retinal persistence (e.g., Brockmole et al., 2002; Di Lollo \& Dixon, 1988; see also Zhang \& Luck, 2008; Ye et al., 2017, 2021). In line with this evidence, previous studies delivered TMS up to $200 \mathrm{~ms}$ after stimulus offset for testing the effects of sensory visual cortex TMS during VSTM encoding and consolidation, and for the effects of TMS on the maintenance phase of VSTM, TMS was delivered at least $200 \mathrm{~ms}$ after stimulus offset (de Graaf et al., 2014; Kammer, 2007; Masse et al., 2020; Serences, 2016; Shevlin, 2020; Xu, 2017, 2020, 2021; see also Brockmole et al., 2002; Di Lollo \& Dixon, 1988; Ye et al., 2017, 2021). In line with this separation in previous TMS studies (Rademaker et al., 2017; van de Ven et al., 2012; van Lamsweerde et al., 2017) and the evidence that consolidation processes might still take place for up to $200 \mathrm{~ms}$ after stimulus offset (Brockmole et al., 2002; Di Lollo \& Dixon, 1988; Jolicoeur \& Dell'Acqua, 1998; Vogel et al., 2006; see also Ye et al., 2017, 2021), we grouped studies inducing TMS for up to $200 \mathrm{~ms}$ after stimulus offset in the VSTM encoding/consolidation phase (from here on referred to as encoding) and studies inducing TMS at least $200 \mathrm{~ms}$ after stimulus offset in the VSTM maintenance phase.

To test the hypothesis that the sensory visual cortex is a necessary component of the brain network that underlies the short-term maintenance of visual information, we performed, to the best of our knowledge, the first systematic review of the TMS literature and the first meta-analyses of this literature. Our specific aims were firstly to systematically collect and appraise the studies that have investigated the role of the sensory visual cortex in the encoding and maintenance of a delayed estimation or a change detection VSTM task using TMS, in order to provide an interpretation of the disparate results. Our second aim was to quantitively synthesize the findings of those studies using meta-analytic methods.

Specifically, two meta-analyses were conducted. The aim of the first meta-analysis was to assess the size of the TMS effect during the encoding VSTM phase, whereas the second meta-analysis aimed to explore and quantify the presence of an effect during the maintenance VSTM phase and compare it to the encoding effect. Additionally, heterogeneity between the identified studies and small study bias was explored. Further, because of the variety of the timings that TMS was induced during VSTM in the included studies, we performed two meta-regressions to explore whether TMS timing (1) after stimulus offset and (2) after stimulus onset were correlated to the TMS effect. Even though meta-analytic methodology is 
more common in clinical research, guidelines have been recently proposed for implementing meta-analyses for basic scientific questions (Mikolajewicz \& Komarova, 2019).

\section{Methods}

\section{Study selection}

A systematic search of three databases (PubMed, Scopus, Web of Science) was conducted to identify relevant papers, according to published guidelines (Mikolajewicz \& Komarova, 2019; Moher et al., 2009; Page et al., 2021). Since this was the first attempt to systematically gather such evidence, the search was conducted without chronological limitations and applied within all fields of the databases. Three of the authors (PP, AT, NK) designed and completed the search strategy.

Initially, data were extracted by a literature search that was conducted in June 2020. The literature search was repeated in March 2021, and one additional study was identified and included in the systematic review and meta-analyses. Details of the final literature search are presented using a PRISMA flow diagram in Figure 1. The literature search was conducted using the following thread: ((("visual short term memory" OR "vstm" OR "visual working memory" OR "short term memory" OR "working memory”)) AND (“primary visual cortex" OR "sensory recruitment" OR "sensory recruitment hypothesis" OR "early sensory cortex" OR “early visual cortex”)) AND (“transcranial magnetic stimulation” OR "tms" OR "behavioural” OR "behavioral” OR "brain stimulation” OR "visual mask” OR "manipulation" OR "reaction time" OR "reaction times" OR “accuracy”). Additionally, studies were identified through previous review papers (Awh \& Jonides, 2001; Christophel et al., 2017; D’Esposito, \& Postle, 2015; Serences, 2016; Sreenivasen et al., 2014; Tapia \& Beck, 2014; van de Ven \& Sack, 2013; Xu, 2017, 2020). Two of the authors (PP and AT) independently assessed the reports that derived from the initial search, based on predetermined inclusion and exclusion criteria (see below). In the case where consensus was not reached between the two, the author NK independently assessed the ambiguous report and group discussions were held to reach a final agreement.

Following the PRISMA statement (Moher et al., 2009) for systematic reviews and meta-analyses, 14 articles matched the criteria and were included in the systematic review. Of these 14 articles, seven provided sufficient statistical data to estimate effect sizes and thus be included in the meta-analyses (see Figure 1). The corresponding authors of the remaining 
papers were contacted through email and further data were requested in order to compute effect sizes and therefore make the studies eligible for the quantitative analysis of this review. One author responded by providing additional data and thus making the final number of included studies in the meta-analyses eight.

\section{Inclusion and exclusion criteria}

To identify papers eligible for the systematic review, three inclusion criteria were determined, which comprised of: (1) behavioral measures of VSTM performance (i.e., accuracy, absolute error, percent correct, precision, guess rate and signal detection), (2) causal interference of the sensory visual cortex using TMS during a VSTM task, and (3) human participants. In addition, two exclusion criteria were defined which included: (1) any form of mental or physical pathology and (2) reports written in a language other than English. No age limitations were set for our search, however the identified studies solely included adults. In order for the studies to be further included in the meta-analyses, they further had to provide arithmetic data (means and SDs or $t$ scores) on behavioral performance scores during a VSTM task in a TMS interference condition and at least one control condition. These variables varied according to study design (i.e., different measures for delayed estimation tasks than for change-detection tasks) and included measurements of accuracy, absolute error, percent correct, precision, guess rate and signal detection $\left(A^{\prime}\right)$.

\section{Data Analysis}

Effect sizes were calculated as the standardized difference between behavioral measures (i.e., accuracies, guess rates, precision, or signal detection) of the experimental condition (i.e., where TMS was induced in the corresponding V1 or V5/MT+ as reported by the authors) and the control condition (i.e., sham TMS, no TMS, weak TMS, or TMS administered to an irrelevant brain area as reported by the authors).

Eight studies provided sufficient statistical data to be included in the meta-analyses. Two meta-analyses were performed: (1) one for experiments inducing TMS during VSTM encoding and (2) one for experiments inducing TMS during VSTM maintenance.

Due to methodological differences between TMS studies (see de Graaf, \& Sack, 2011), significant heterogeneity, as indicated with the $I^{2}$ index was expected between the studies (van de Ven \& Sack, 2013). According to Higgins, Thompson, Deeks, and Altman (2003), the $I^{2}$ index levels can be described as low, moderate, and high, when they fall close to $25 \%, 50 \%$, and $75 \%$, respectively. We estimated effect sizes for each individual data set 
using Hedge's $g$ formula (Hedges, 1981). In order to quantify the overall effect size of TMS, we used the absolute values of the effect sizes in our meta-analysis models (see Fritz et al.,2012; Morrissey, 2016), which has several advantages compared to the use of signed effect sizes. Specifically, absolute effect sizes avoid alternative explanations for the inhibitory and facilitatory TMS effects that were identified (see section Systematic review summary) and cannot be explored due to the small number of the included studies that restrict us from exploring moderator effects in the analyses. Further, because the effect sizes were calculated from different types of behavioral measures, using the signed effect sizes would require reversing the signs of some effect sizes so that they all point towards enhancement or inhibition. For example, an effect size greater than 0 calculated from guess rates indicates a performance drop, while a similar effect size calculated from percent correct shows performance increase. Moreover, using the signed effect sizes may lead to bias in the conclusions. As an example, in studies that compare VSTM performance in contralateral versus ipsilateral sensory visual cortex TMS it is unclear if a difference in performance reflects TMS inhibition or facilitation. For example, a study that treated TMS over the contralateral sensory visual cortex as the experimental condition and TMS over the ipsilateral side as the control condition will interpret a performance drop (e.g., contralateral accuracy < ipsilateral accuracy) as an inhibitory TMS effect. However, given recent evidence supporting the role of the ipsilateral sensory visual cortex in visual processing (Zhao et al., 2021) and the visual pathway neuroanatomy (see Joukal, 2017; Wichmann \& Müller-Forell, 2004), it could be reasonable to assume that the ipsilateral sensory visual cortex is in fact the experimental condition. In such a case, the conclusion of the same study, with a different definition of the experimental and control conditions might turn out to be the opposite (e.g., facilitation effects since ipsilateral accuracy $>$ contralateral accuracy). We therefore opted to use the absolute values of the effect sizes which were then pooled using a random-effects model (Fleiss, 1993).

Data analysis was conducted using R (v4.0.2) and Rstudio (v1.1.456) (R Core Team, 2020; see also Harrer et al., 2019). Hedge's g (Hedges, 1981) effect sizes were calculated using the esc library (Lüdecke, 2018). The effect sizes were pooled using a random effects model (Fleiss, 1993) using the meta package for R (Schwarzer, 2007). Specifically, the metaanalyses were conducted using the inverse variance method, where variance includes both within- and between-study variance. The maximum-likelihood estimator was used for tau ${ }^{2}$ and the $Q$-profile method was used for the tau and tau ${ }^{2}$ confidence intervals. Forest plots were used for data visualization. Due to the small number of studies included in the meta- 
analyses, moderator variables analysis was not possible to conduct. To test for small study bias, funnel plots were generated to visually investigate their symmetry which was further examined using the Egger's test (Egger et al., 1997). Mikolajewicz and Komarova (2019) provide a comprehensive summary for how Hedge's $g$, tau ${ }^{2}, Q$, and $I^{2}$ are formulated.

Some individuals participated in more than one experiment and/or experimental condition. Because this violates the independency of some data points, we performed a threelevel meta-analysis (see Assink \& Wibbelink, 2016; Cheung, 2014; Pastor \& Lazowski, 2018). We included an additional level in the meta-analyses, referred here as the study level, where we clustered each experiment and/or experimental condition to its corresponding study. This analysis allowed us to explore how the different levels explain variance in the model. We then repeated the analyses excluding the study level and compared the fitness of the two-level and three-level models.

Lastly, since the timing of the induced TMS varied between the included studies, we performed two meta-regressions on the calculated effect sizes using the TMS timing point of each effect size as a covariate; one for TMS timing after stimulus offset, and one for TMS timing after stimulus onset. These meta-regressions served a double purpose: (1) explore whether stimulation timing can predict the TMS effect and (2) explore whether the TMS effect differs according to the stimulation timing without categorizing it in one of the two VSTM phases.

\section{Data availability statement}

All relevant data used in the study are openly available and can be accessed on https://osf.io/p8nwz.

\section{Results}

The systematic search of the literature led to the identification of 14 papers. These papers included a total of 18 experiments that interfered with sensory visual cortex activity using TMS during a VSTM task. A total of 248 individuals participated in the 18 experiments. Short descriptions of the included studies and their experiments are presented in Table 1. Six of these papers were excluded from the meta-analysis due to a failure to report sufficient statistical data (Cataneo et al., 2012; Malik et al., 2015; Saad et al., 2015; Silvanto \& Cattaneo, 2010; Silvanto \& Soto, 2012; Soto et al., 2012).

\section{Systematic Review}


Methodological Issues. A number of methodological issues such as the different apparatuses used (i.e., stimulator, coil), targeting methods (e.g., neuronavigation, phosphene induction), as well as the different output settings (e.g., power, frequency, number of pulses) have been identified (de Graaf, \& Sack, 2011; Pitcher, et al., 2020; Sadrini, et al., 2011) as factors that can possibly affect the homogeneity of the experiments (van de Ven \& Sack, 2013). In the studies considered here, TMS stimulation was delivered with a $70 \mathrm{~mm}$ figure-of-eight coil in all experiments. The majority of experiments $(n=16)$ targeted area V1, while two focused on $\mathrm{V} 5 / \mathrm{MT}+$. Eight of the included experiments aimed to directly investigate the role of the sensory visual cortex in VSTM. The remaining six studies had different aims, but nevertheless reported behavioral outcomes whilst interfering with TMS on the sensory visual cortex during a VSTM task, making them useful for the purposes of this systematic review and meta-analysis. In eight of the included experiments, TMS output power was determined using the functional method of eliciting phosphenes (see Walsh, \& Pascual-Leone, 2003), while in the remaining ten a fixed TMS power output was used. When interfering with the sensory visual cortex, two experiments delivered TMS in four pulses, four in five pulses, six delivered three-pulse TMS, and six experiments delivered a single TMS pulse. Moreover, eight experiments targeted the sensory visual cortex in only one hemisphere.

In all experiments, control conditions were used in order to compare with possible effects in the TMS conditions. These controls differed between the experiments. Despite the fact that in seven experiments there were control conditions where no TMS was applied at all, other control conditions were also included to account for the noise and haptic artefacts of the stimulation. In particular, within these seven experiments, three additionally compared sensory visual cortex stimulation with vertex stimulation. The remaining four stimulated only one hemisphere and therefore used the ipsilateral -to the stimulation region of interest (ROI)visual hemifield condition as a control (compared to the contralateral one). In two experiments, an ipsilateral visual hemifield condition was used as the only control, while in six experiments control was solely a sham TMS condition. One experiment used both an ipsilateral visual hemifield and sham TMS condition as controls. In the remaining two experiments, one used a low (ineffective) TMS output power as a control, while the other used both low powered TMS and vertex TMS.

The inconsistency between the methods used leads to two important issues. Firstly, the lack of a specific TMS protocol to be followed in a certain field of research can produce mixed or misinterpreted results (de Graaf, \& Sack, 2011; Sadrini, et al., 2011). Second, it does not support reproducible science, and in the case of a meta-analysis, could lead to 
significant heterogeneity (see van de Ven \& Sack, 2013). Taken together, these findings highlight the wide variability of methods (e.g., stimulation parameters) used to study TMS interference as an important factor in why the role of sensory visual cortex in VSTM still remains unclear.

TMS interference during encoding. The effects of TMS interference of the sensory visual cortex activity during the encoding phase of visual information was tested in six experiments, from five studies (Cattaneo et al., 2009; Koivisto et al.,2017; Rademaker et al., 2017; van de Ven et al., 2012; van Lamsweerde, \& Johnson, 2017). As expected, the majority of these studies presented evidence supporting the involvement of the sensory visual cortex during VSTM encoding.

Cattaneo et al. (2009) used TMS to interfere with sensory visual cortex during a visual imagery task or a VSTM task. Participants were presented for 1 second with either an analog time which they had to remember (VSTM task) or a digital time, of which they had to imagine and remember the analog form (visual imagery task). Stimulation was applied at 0 $\mathrm{ms}$ after stimulus offset, at the beginning of a 2 second delay period, which is typically considered the encoding phase of VSTM (Bays et al., 2011; Brady et al., 2016; Kammer, 2007; Vogel et al., 2006). Participants then had to respond whether a dot would fall within or outside the remembered clock-hands. Accuracy and reaction times were compared between the sensory visual cortex TMS condition, a no-TMS control condition, and a vertex TMS control condition. Reaction times were significantly slower during the sensory visual cortex TMS conditions in the VSTM task, compared to the two controls, indicating an impairment in the performance of a VSTM task as a result of TMS during the encoding phase, therefore supporting the involvement of the sensory visual cortex in VSTM encoding.

Subsequent studies reported experiments that provide further evidence for the involvement of the sensory visual cortex in VSTM encoding using more sensitive statistical methods, such as mixture models (see Grange et al., 2021). Koivisto et al. (2017) conducted two experiments to investigate whether TMS affects precision or guessing rates in a VSTM task, and whether these are affected dichotomously ('all or nothing') or gradually. The orientation of a remembered Landolt-C presented for $12 \mathrm{~ms}$ (or $24 \mathrm{~ms}$ for $n=1$ ) had to be matched to a probe presented after a 1 second delay period. In the first experiment TMS was delivered at $-30,0,30,60,90$, and $120 \mathrm{~ms}$ relevant to the onset of the stimulus and in the second experiment at 120,150, and $180 \mathrm{~ms}$ after stimulus onset. The proportion of guess trials and the precision in the task were compared between the ipsilateral and contralateral conditions, as well as with a no-TMS control condition. The results showed higher guessing 
rates across both experiments in the contralateral TMS condition between 60 and $150 \mathrm{~ms}$ demonstrating the effects of TMS interference in the sensory visual cortex during the encoding phase of VSTM.

Koivisto et al. (2017) provided evidence supporting the involvement of the sensory visual cortex during VSTM encoding through their paradigm, even though their research objectives differed from the ones of this systematic review. Following these sensitive statistical methods, some studies, which directly investigated the role of the sensory visual cortex during VSTM encoding, provide additional support for its involvement. Rademaker et al. (2017) asked participants to match the orientation of one out of four gratings which were presented for $200 \mathrm{~ms}$ in four visual field quadrants corresponding to either the same, ipsilateral, contralateral, or diagonal TMS ROI. TMS was delivered either at the beginning ( 0 ms after stimulus onset) or midway during a 2 second delay period (900 ms, see section TMS interference during maintenance for further details) of the VSTM task and a sham TMS condition was also used as control. The authors reported more errors when stimulation matched the remembered-item location compared to when the remember-item location was furthest to the stimulation. Also, early stimulation had a significantly stronger effect compared to late stimulation. Additionally, higher precision was measured when the pulse and target overlapped (same and ipsilateral conditions) compared to when they were far apart (diagonal condition) and guessing was reported higher when TMS was earlier than later.

Similarly, participants in an experiment by van Lamsweerde and Johnson (2017) had to remember the color of three squares presented for $150 \mathrm{~ms}$. A probe presented after a 1 second delay period asked them to match the color of one of the three remembered squares. During the VSTM task, TMS was induced at 0, 100, or $200 \mathrm{~ms}$ after stimulus offset. Their results indicated that guess rates were higher when TMS was applied at stimulus onset in the contralateral condition. Swap rate effects (an indication of recalling a non-cued item) were also reported, which were significantly decreased in the contralateral compared to the ipsilateral condition. Further, a significant interaction of TMS and side (ipsilateral/contralateral) was reported on precision, with the effect seeming stronger at earlier TMS timing conditions. Given the stronger effects at earlier TMS timing conditions, similar to Koivisto et al. (2017) described above, the authors concluded that TMS effects are evident during memory encoding but are no longer effective once consolidation in VSTM has been achieved.

Van de Ven et al. (2012), found no effect on the performance in a VSTM task when sensory visual cortex TMS interfered during encoding at $100 \mathrm{~ms}$ after stimulus onset but did 
find an interference effect at $200 \mathrm{~ms}$ post stimulus onset. Participants performed a change detection task on a sample of either one (low load condition) or three (high load condition) non-natural shapes presented for $150 \mathrm{~ms}$ and maintained in VSTM during a 1.5 second delay period. Participants had to respond whether a probe was the same or different as the memory sample and accuracy of change detection was measured using signal detection theory. During the delay period, TMS was induced at $100 \mathrm{~ms}, 200 \mathrm{~ms}$, or $400 \mathrm{~ms}$ after the memory set onset. A significant effect was found only in the $200 \mathrm{~ms}$ high load condition (described further in the TMS interference during maintenance section), which led the authors to the conclusion that the sensory visual cortex's involvement in VSTM mainly takes place during the early maintenance phase.

In summary, the findings of the studies described above indicate that, apart from the experiment reported by van de Ven et al. (2012), all five remaining experiments reported a TMS effect on behavioral performance during the encoding phase of a VSTM task indicating that the results from studies using TMS to interfere with the sensory visual cortex during the encoding phase of VSTM are consistent with the well-established role of the sensory visual cortex in VSTM encoding. Next, we turn to the evidence from human TMS studies examining the involvement of sensory visual cortex in short-term maintenance.

TMS interference during maintenance. TMS was delivered during the maintenance phase of a VSTM task (i.e., more than $200 \mathrm{~ms}$ after stimulus onset) in 14 experiments from twelve different studies (Cattaneo et al., 2012; Jia et al., 2021; Malik et al., 2015; Rademaker et al., 2017; Saad, \& Silvanto, 2013; Saad et al., 2015; Silvanto \& Cattaneo, 2010; Silvanto, \& Soto, 2012; Soto et al., 2012; van de Ven et al., 2012; van Lamsweerde, \& Johnson, 2017; Zokaei et al., 2014). As discussed in detail below, although the majority of these studies provide evidence supporting the sensory recruitment hypothesis, others either failed to find such evidence or their findings are more consistent with an interaction between perceptual and VSTM processes, as we suggest below.

Silvanto and Cattaneo (2010) investigated the role of the motion selective V5/MT+ area in VSTM. The VSTM task consisted of two successively presented moving stimuli, which had either a right or a left direction and were presented at two different speeds. In each condition, the two stimuli were presented for $300 \mathrm{~ms}$ each and moved at different directions, but at the same speed. A cue followed the stimuli presentation and informed participants which of the two stimuli should be maintained; 3 seconds into the 5.5 second delay period, TMS was delivered to area V5/MT+. Next, participants had to subjectively rate the location 
and direction of movement of the phosphene before being presented with a probe stimulus. The probe had the same direction as the memory sample and participants were asked to report whether the speed of the last motion stimulus was faster or slower than the memory sample. The authors reported that when moving phosphenes overlapped and had the same direction as the moving stimuli, memory accuracy was significantly lower compared to the no TMS condition and higher compared to when phosphenes overlapped but moved in the opposite direction to the stimuli. These results indicate that, further to the involvement of the sensory visual cortex during VSTM maintenance, area V5/MT+ maintains visual memory representations in a retinotopic manner.

The involvement of area V5/MT+ in VSTM maintenance was further supported in two experiments by Zokaei et al. (2014). In the first experiment participants were asked to remember two motion stimuli presented above and below fixation for $200 \mathrm{~ms}$. The color of each stimulus was either red or green and one color was probed. Participants had to match the movement direction of the remembered stimulus to that of the probe. During the 3.7 second delay period of this task, participants were also asked to identify, after being probed with a color, if the same-colored stimulus was above or below fixation. After 3.2 seconds into the delay period, TMS was delivered, followed by the memory probe. In the second experiment, the two motion stimuli, instead of being presented together, were sequentially presented for $300 \mathrm{~ms}$ each and each was followed by a 1 second delay period. TMS was applied $300 \mathrm{~ms}$ after the onset of either the first or the second stimulus. Following the second stimulus, a colored probe appeared indicating to participants to match its direction to that of the samecolored motion stimulus's direction. A low intensity TMS condition was used in both experiments as a control condition and a vertex TMS condition was additionally introduced in the second experiment. The results of the first experiment showed that in the ineffective TMS condition there was a significant impairment in performance between congruent (if the position identification task probe matched the color of the memory task probe) and incongruent (if the position identification task probe did not match the color of the memory task probe) conditions which disappeared in the effective TMS condition. Similarly, in the second experiment, a significant impairment in precision was found in the low TMS condition when the first stimulus was followed by TMS, compared to when the second stimulus was followed by TMS; this effect was not evident in the high TMS condition. The authors described this as a facilitation effect of TMS, explaining that non-privileged memory items (i.e., memories in the incongruent trials; see Hitch et al., 2020) were likely suppressed and thus enhanced by stimulation. Since TMS can enhance neural excitability, in addition to 
inhibiting brain processing (Robertson et al., 2003), this facilitation effect is consistent with the findings of Silvanto and Cattaneo (2010) suggesting the involvement of area V5/MT+ during VSTM maintenance.

In addition to the involvement of area V5/MT+, TMS evidence for the involvement of early visual areas V1/V2 was provided in the studies discussed below. Cattaneo et al. (2012) applied TMS on area V1 of the sensory visual cortex during the delay period of a VSTM task. In the task, participants were presented with a digital time for 1 second and had to remember the equivalent analog clock-hands. At the beginning of each trial, an adaptor was used that either overlapped with the to-be-remembered clock-hands or not. TMS was delivered 1 second after stimulus onset and at the end of a $700 \mathrm{~ms}$ delay period, participants responded whether a dot fell within or outside the remembered clock-hands. Results of the participants' accuracies indicated that the adapter decreased performance in the no-TMS and vertex-TMS control conditions, but the adapter's effect disappeared in the sensory visual cortex TMS condition. Similar to Zokaei et al. (2014), a facilitation effect of the TMS was found, which suggests that, similarly to area V5/MT+, early visual areas V1/V2 are also involved in VSTM maintenance.

A recent study by Jia et al. (2021), studying the effects of perceptual training in VSTM, provided strong evidence in support of the sensory recruitment hypothesis for area V1. Specifically, they used an orientation change detection task, where participants had to remember the orientation of one grating presented for $200 \mathrm{~ms}$, and report whether a probe presented after a $4 \mathrm{~s}$ delay period had a clockwise or counterclockwise tilt compared to the remember stimulus. TMS was applied $1.5 \mathrm{~s}$ into the delay period. Participants completed the task twice, once before and once after perceptual training. In both cases, TMS significantly impaired accuracy in the VSTM task.

Additional evidence for the role of areas V1/V2 was found by van de Ven et al. (2012) (also described above in the section TMS interference during encoding), during sensory visual cortex stimulation in two different conditions throughout the 1.5 second delay period, at $200 \mathrm{~ms}$ and $400 \mathrm{~ms}$ after stimulus onset (as well as at $100 \mathrm{~ms}$ corresponding to encoding; discussed in the previous section). TMS affected task performance in the contralateral compared to the ipsilateral condition only in the high load $200 \mathrm{~ms}$ TMS condition. These findings support the involvement of the sensory visual cortex in the maintenance of visual information, mainly during the $200 \mathrm{~ms}$ window. Likewise, in Rademaker et al.'s (2017) study (also described previously; see TMS interference during encoding section), the sensory visual cortex was stimulated $900 \mathrm{~ms}$ into the delay period of 
their VSTM task. As discussed previously, more errors were reported when stimulation matched the remembered-item location compared to when the remember-item location was furthest to the stimulation and higher precision was reported when TMS and target overlapped (same and ipsilateral conditions) compared to when they were further apart (diagonal condition). Taken together these results indicated that stimulation that overlapped with the same or ipsilateral visual field affected task performance when compared to the visual field that was further apart. However, it should be noted that these results were larger for earlier (during encoding) rather than later (during maintenance) stimulation. Similar evidence was shown in van Lamsweerde and Johnson's (2017) work (also discussed previously; see TMS interference during encoding section), whose results showed a significant interaction of TMS and side (ipsilateral/contralateral) on precision, with the effect seeming stronger at the $100 \mathrm{~ms}$ after stimulus offset TMS timing condition. On the same line with van de Ven et al. (2012) and Rademaker et al. (2017), van Lamsweerde and Johnson's (2017) effects were stronger during their $0 \mathrm{~ms}$ and $100 \mathrm{~ms}$, rather than their $200 \mathrm{~ms}$ condition, suggesting that the sensory visual cortex is involved in the earlier stages of VSTM.

Further to the inhibitory and facilitatory effects of TMS during VSTM maintenance, two studies discussed an interaction of TMS between perceptual and memory processes. Silvanto and Soto (2012) studied the intervention of subliminally perceived visual items in the sensory visual cortex. In Experiment 1b TMS was applied over the sensory visual cortex during a VSTM task. Participants were instructed to remember the orientation of a grating presented for $200 \mathrm{~ms}$ over a 2 second delay period. In the majority of the trials (66\%), a low contrast distractor appeared 1 second into the delay period for $13 \mathrm{~ms}$, which was either congruent (same) or incongruent (different) from the memory sample. TMS was also induced 1 second into the delay period and sham TMS was used as control. The results indicated that when there was no distractor present (remaining 34\% of trials), sensory visual cortex TMS impaired the ability to detect the probe difference but facilitated this ability when the distractor was incongruent compared to the sham TMS condition. The authors explained these results as a possible perception and memory mechanisms interaction, where TMS possibly enhanced neurons in a suppressed state at the incongruent distractor condition, thus making it easier to perceive. These results are in line with work indicating the statedependency of TMS effects, which show that neural activity before stimulation can modulate the effects of stimulation (Bestmann et al., 2007; Pasley et al., 2009; Silvanto et al., 2018; for a recent review see Silvanto \& Cattaneo, 2017; see also Discussion). 
In a similar manner, an interaction between perception and memory processes was discussed by Saad et al. (2015), who investigated the differences between imagery and VSTM neural bases. In their VSTM condition, participants had to memorize the contrast of a grating presented for $300 \mathrm{~ms}$ throughout a 4 second delay period. Sensory visual cortex TMS was applied 2.6 seconds into the delay period, and the delay was followed by a probe grating for which participants had to indicate whether it had a higher or lower contrast compared to the remembered one. The probe could either be slightly or more noticeably different in contrast than the remembered, thus introducing a harder or easier condition, respectively. A sham TMS condition was used to allow for comparisons. Additionally, during different blocks of the experiment, participants were asked to either create a mental image of the remembered stimulus (imagery condition) or not (VSTM condition). Results indicated that sensory visual cortex TMS enhanced detection sensitivity relative to sham TMS in both the imagery and VSTM conditions. However, when it came to reaction times, sensory visual cortex TMS only had an effect in the VSTM condition compared to sham, where reaction times were found to be slower. In line with previous studies (Cattaneo et al., 2012; Zokaei et al., 2014), the enhancement of detection sensitivity by stimulation in the VSTM and imagery tasks were discussed as TMS facilitatory effects. The difference found in reaction times between the VSTM and imagery conditions, was attributed to perceptual processes, where in the memory condition, the noise added by TMS possibly affected the time needed to gather perceptual evidence to judge in the discrimination task.

Silvanto and Soto (2012) and Saad et al. (2015), further to supporting the involvement of the sensory visual cortex in VSTM maintenance, reported an interaction between perception and VSTM. A similar interaction was noticed in two other studies, despite the fact that no other inhibitory or facilitatory TMS effects were found to support the involvement of the sensory visual cortex in VSTM maintenance. Soto and Silvanto (2012) combined a priming task with a memory task in order to investigate attentional guidance. Participants were cued whether they should remember (VSTM task) or just look (priming task) at a colored circle, which was presented for $200 \mathrm{~ms}$. In the VSTM task, after an individually adjusted delay period, a probe appeared and participants had to respond whether it was the same or different circle as the memory sample one. TMS was delivered at area V1 1 second after the memory sample onset. At 1 second during the delay period, along with the TMS, a search task asked participants to identify which of two circles had a horizontal gap and report whether the gap was on the left or right side. In the priming task, no memory probe was shown after the search task. No effects on VSTM performance were found between the TMS 
and a sham-TMS condition but the effects of TMS for the priming search task were significantly modulated by memory requirement. Specifically, participants responded more accurately in the search task in the TMS condition but only when memory was required. In line with the previously mentioned studies (Saad et al., 2015; Silvanto \& Soto, 2012) these effects indicate an interaction of TMS with perceptual processes, modulated by what is maintained in memory.

Similar results were found in a group of experiments by Saad et al. (2013), where they examined how the tilt aftereffect can affect memory representations. In two of their experiments, sensory visual cortex TMS was applied during a VSTM task. In the VSTM condition of the first experiment participants were requested to remember the orientation of a grating and in the second experiment the color and size of one square. In both cases, the memory sample was presented for $300 \mathrm{~ms}$ and was maintained during a 5.3 second delay period, which was followed by a same-sized adapter grating, that had either the same or a 20degree tilt difference (in the same direction) from the memory sample. In the first experiment, TMS was delivered either at 2 or 5 seconds into the delay period. In the second experiment, TMS was delivered only at 2 seconds into the delay period. In the VSTM conditions, a probe appeared after the delay period where participants had to report the change from the memory sample (first experiment) or match it to the memory sample (second experiment). Results showed no difference between TMS and a sham TMS control condition on the memory task, however the tilt aftereffect, was significantly decreased by TMS in the memory condition (compared to a passive one). Similarly to the results of Soto and Silvanto (2012), the tilt aftereffect, which is a perceptual process, was modulated by memory requirements, indicating once more an interaction between perceptual and memory processes.

Even though the majority of studies showed either a direct TMS effect or a perception and VSTM interaction effect due to TMS, one study failed to find any evidence in support of the sensory recruitment theory. Malik et al. (2015) investigated the role of the sensory visual cortex across trans-saccadic remembered features and used a VSTM TMS paradigm in one of their experiments. Participants had to remember the orientation of one grating presented for $100 \mathrm{~ms}$ and then report the difference in direction between the remembered one and a probe grating (i.e., clockwise or anticlockwise). TMS was induced $200 \mathrm{~ms}$ after the start of a 900 ms delay period. Comparisons were made between the contralateral and ipsilateral TMS ROI to visual hemifield condition, as well as in comparison to a no TMS condition. No differences were found, which, according to the authors, is consistent with previous evidence that TMS 
delivered over the sensory visual cortex does not interfere in low VSTM load conditions, for example, when only one item has to be maintained (van de Ven et al., 2012).

Taken together, the results from the systematic review on TMS interference during memory maintenance indicate that the sensory visual cortex is likely involved in VSTM maintenance, supporting the sensory recruitment hypothesis. This is reflected in all but one of the studies by the direct TMS effects on memory performance and the TMS interaction effect between perception and VSTM. Following the description of the included studies, below, we summarize the results of the systematic review regarding the role of the sensory visual cortex in VSTM.

Systematic review summary. The majority of the described studies indicated a likely involvement of the sensory visual cortex in both the encoding and maintenance phase of VSTM. However, the results provided by the identified studies, especially those applying TMS during the maintenance phase of VSTM, reflect some issues likely deriving from the variety of methodological approaches used between the studies.

Regarding VSTM encoding, all but one study (van de Ven et al., 2012) provided evidence in support of the role of the sensory cortex. The lack of such an effect in the $100 \mathrm{~ms}$ condition of the van de Van et al. (2012) could be due to a number of methodological issues. Specifically, van de Ven et al. (2012) used two different localisation methods and found a significant difference in the variable of interest (significantly different $A$ ' under the higher load condition of their experiment, which was the only condition reported to have significant results) between the participants depending on which localisation method group they belonged to. Furthermore, since comparisons were made between the memory load condition and the TMS timing, it is likely that a TMS effect actually does exist in both conditions (i.e., TMS affected memory performance in both load conditions). For example, no additional control condition (e.g., sham or no TMS) was used other than the ipsilateral visual hemifield of the targeted ROI. Therefore, because of the lack of dichoptic stimulus presentation (see Carmel et al., 2010), it remains possible that encoding of the visual information was in fact processed by the sensory visual cortex in both hemispheres (Tong et al., 2006; Zhao et al., 2021) and thus the effect remained undetected when comparing the ipsilateral versus the contralateral condition of the experiment.

Another issue reflected in the results of the included studies, concerns the fact that some studies provided evidence of inhibitory TMS effects (Jia et al., 2021; Rademaker et al., 2017; Silvanto \& Cattaneo, 2010; van de Ven et al., 2012; van Lamsweerde, \& Johnson, 
2017), while others reported facilitatory TMS effects (Cattaneo et al., 2012; Saad et al., 2015; Zokaei e al., 2014) on VSTM performance. Examining the methodological differences between these studies, a possible explanation of this contradiction could lay in the distinct stimulation power output used for TMS. Specifically, it has been reported that lower TMS outputs can often lead to facilitation effects, while suprathreshold intensities are needed in order to disrupt activity and behavior (Kim et al., 2015; Moliadze et al., 2003; Silvanto et al., 2018; see also Silvanto \& Cattaneo, 2017). In the current systematic review, studies showing facilitation effects utilized a standard TMS output for the majority of their participants, while the rest used an individualized threshold. Alternatively, the mixed inhibitory and facilitatory TMS effects might unveil a different TMS interference effect between distinct storage mechanisms. For example, TMS could in some cases interfere with active neural representations, thus inhibiting performance, whereas in other cases TMS can facilitate activity that is close to baseline (see Robertson et al., 2003; Rose et al., 2016; Silvanto \& Cattaneo, 2017). In line with this alternative explanation, it has been recently postulated that VSTM might employ a variety of processes to protect visual representations (Lorenc et al., $2018,2020)$ and that these contradictory TMS effects might reflect the use of different storage mechanisms (Adam et al., 2021; Silvanto \& Cattaneo, 2017).

The majority of the TMS studies investigating the involvement of the sensory visual cortex during VSTM maintenance reported evidence supporting the sensory recruitment hypothesis. However, four experiments described in three different papers reported no direct significant effects of sensory visual cortex TMS on memory performance (Malik et al., 2015; Saad, \& Silvanto, 2013; Soto \& Silvanto, 2012). A closer look at the experiments that did not report any TMS effects unveils an interesting common denominator. Specifically, all four experiments presented only one stimulus that was ought to be remembered. In fact, in the fixation task experiment by Malik and colleagues (2015), which was designed as a control condition for their main research objectives, the authors reported that no significant results were expected, since sensory visual cortex TMS in such low load conditions has been previously found to be ineffective (van de Ven et al., 2012). In the two experiments reported by Saad et al. (2013), no effect was found when sensory visual cortex TMS was compared to a sham condition. Though, further analyses indicated that TMS was more effective during the VSTM task when compared to a passive condition (with no memory maintenance requirement). Likewise, no effects were reported in the work of Soto and Silvanto (2012) on VSTM performance between the TMS and sham conditions. However, effects by sensory 
visual cortex TMS in their priming task, were significantly modulated by whether memory maintenance was required or not.

This interaction between perceptual and memory mechanisms, as well as the role of processing load, have been identified and reported in other sensory visual cortex TMS studies (Saad et al., 2015; Silvanto \& Soto, 2012; van de Ven et al., 2012). This interaction has also been supported by behavioral studies, which suggested that the perception of visual stimuli and VSTM are underlaid by shared neural mechanisms (Magnussen et al., 1991; McKeefry et al., 2007). Furthermore, the VSTM load and perceptual performance relationship has been previously studied, indicating that VSTM capacity load can affect both performance and sensory visual cortex activity (Konstantinou, et al., 2012, 2014; Konstantinou, \& Lavie, 2013, 2020). In fact, the sensory recruitment hypothesis stems from this shared neural substrate between perception and VSTM (Pasternak, \& Greenlee, 2005), which is reflected in dual and distraction tasks (for a recent review see Lorenc et al., 2021). Thus, a possible explanation for the failure to detect a TMS effect in these experiments is low sensory visual cortex neural demands (i.e., perceptual and/or memory load). For example, studies have indicated that the maximum number of visual objects that can be maintained in VSTM is estimated to range between three and four items (Cowan et al., 2005; Luck, \& Vogel, 1997; Todd, \& Marois, 2004; Vogel, et al, 2001, 2005; Vogel, \& Machizawa, 2004). Therefore, if this is reflected in the sensory visual cortex's activity, it is possible that enough resources were still available in the sensory visual cortex due to low load VSTM task demands, thus, TMS noise leaves the maintained representations unaffected (see de Graaf, \& Sack, 2011). It could be argued that in some cases, where only one stimulus was presented, a strong TMS effect was found (Jia et al., 2021). However, some methodological differences could explain why Jia et al. (2021) were able to show an effect despite the sensory load. Specifically, in the Jia et al. (2021) study, an orientation stimulus combined with retinotopic mapping was employed, which allowed researchers to identify and stimulate specific V1 areas which correspond closely to the neurons processing the stimulus orientation.

Additionally, another methodological issue that likely contributes to the debate, relates to the stimulus complexity used for the memory array in VSTM tasks. It has been reported that sensory visual cortex is involved in the maintenance of elemental visual features such as orientation and direction of movement (Harrison \& Tong, 2009; Serences et al., 2009). However, when stimuli complexity increases, different brain regions might be recruited for encoding and maintenance, such as the intraparietal sulcus (Xu \& Chun, 2006; $\mathrm{Xu}, 2007$ ) and the posterior parietal cortex (Song \& Jiang, 2006). Therefore, in studies using 
complex stimuli (e.g., van de Ven et al., 2012), the neural processes required for successfully maintaining visual information in VSTM might involve higher order brain areas in addition to sensory visual cortex (Teng \& Postle, 2021). This might result to null effects when TMS is applied over sensory visual cortex during the memory delay since such representations might be protected through a distributed VSTM network (Lorenc \& Sreenivasan, 2021; see also Gayet et al., 2018; Scimeca et al., 2018).

Overall, the systematic review provides additional evidence for the well-established role of the sensory visual cortex in VSTM encoding, by identifying and summarizing the relevant TMS studies. Further, the systematic review of TMS studies supports the involvement of the sensory visual cortex during VSTM maintenance. It is suggested that the contradictory results derive from the variety of methods utilized, such as the binocular presentation of stimuli, the storage mechanism of VSTM representations, the memory and perceptual load, and the memory stimulus complexity. Next, we turn to a quantitative analysis of these results using meta-analytic methodology in order to statistically quantify and test the conclusions of the systematic review.

\section{Meta-Analysis}

Meta-analysis 1: TMS interference during encoding. Five studies totaling $n=204$ participants were included in the meta-analysis investigating the effect of TMS during the encoding of visual information. Out of these five studies, a total of 18 effect sizes were calculated based on all the relevant experimental conditions. All effect sizes are presented in Table 2.

As expected, heterogeneity was significant amongst data sets, $Q(17)=75.67, p<$ .0001 , with high inconsistency between studies, $I^{2}=77.5 \%, \tau^{2}=.80,95 \% C I=.47,2.94$. We proceeded with a random effects model which provided a significant standardized difference in means of $g=.80,95 \% C I=.35,1.25, Z=3.46, p=.0005$, indicating that TMS during encoding on the sensory visual cortex does have a high effect on VSTM behavioral outcomes. The forest plot of the meta-analysis during encoding is illustrated in Figure 2 (top).

Further, small study bias was investigated using visual inspection of a generated funnel plot (see Figure 3A) and using the Egger's Test. The right side of the inverted funnel is underrepresented, indicating that more studies showing a stronger TMS interference effect in VSTM encoding are needed to make the funnel symmetrical. Both approaches indicated possible small study bias, as reflected in the asymmetry of the funnel plot and the significant Egger's Test, intercept $=4.85, t(17)=3.59, p=.002$. 
Meta-analysis 2: TMS interference during maintenance. A total of seven studies totaling $n=$ 206 participants were included in the meta-analysis of the effect of TMS during the maintenance of visual information. From the relevant experimental conditions of these seven studies, a total of 14 effect sizes were calculated. The effect sizes are presented in Table 3.

Heterogeneity was not violated amongst the data sets, $Q(13)=8.23, p=.83$, with no inconsistency between studies, $I^{2}=0 \%, \tau^{2}=0,95 \% C I=0, .05$. Given the methodological differences used in the included studies, this was an unexpected finding. However, it must be noted that the $I^{2}$ confidence intervals were wide $(C I=0 \%, 55 \%)$, thus making any conclusions regarding heterogeneity difficult to reach. The random effects model provided a significant standardized difference in means of $g=.50,95 \% C I=.35, .65, Z=6.51, p<$ .0001 , providing evidence that TMS applied on the sensory visual cortex during the maintenance phase of a VSTM task results in a significant moderate difference on VSTM behavioral outcomes. The forest plot of the second meta-analysis is shown in Figure 2 (bottom).

As previously, small study bias was investigated by visually inspecting a generated funnel plot (see Figure 3B) and with the Egger's Test. No asymmetry was found in the funnel plot, indicating that effect sizes were evenly distributed, as also confirmed by the Egger's Test, intercept $=1.50, t(13)=3.92, p=.30$. Notably, no values seem to be plotted on the top of the horizontal (y) axis of the funnel plot, which represents lower effect size standard error. This likely reflects the lack of studies with large sample sizes, which have greater statistical power and consequently reduce error. However, the lack of asymmetry evidence is possibly explained by the fact that the effect sizes lay between a narrow range of standard error $(0.22$ to 0.40$)$.

Comparison of the two Meta-analyses: In order to compare the two overall effect sizes, the data from both meta-analyses were analyzed together and an overall random effect was pooled for all 32 effect sizes (see Figure 2). The overall test of heterogeneity was significant $Q(31)=84.03, p<.0001$, showing an inconsistency between studies, $I^{2}=63.1 \%, \tau^{2}=0.12$, $95 \% C I=.20,1.56$. The random effects model of all studies indicated a significant moderate effect of TMS on VSTM performance, $g=.58,95 \% C I=.41, .75, Z=6.74, p<.0001$. Further, a comparison between the encoding and maintenance random effects models, showed no significant differences between the two overall effects, $\chi^{2}(1)=1.50, p=0.22$, providing an indication that a sensory visual cortex TMS effect on VSTM performance is similarly evident in both the encoding and maintenance VSTM phases. 
The three-level meta-analysis indicated that no variance was explained by the study level, $\sigma^{2}=0$, total $I^{2}=7.45 \%$ (see Figure 4). An analysis of variance comparison of the twolevel model $(d f=2, A I C=73.77, B I C=76.64)$ and the three-level model $(d f=3, A I C=$ $75.77, B I C=80.07)$ showed no significant differences between them $(p=1)$. Since no variance was explained by the study level, and no significant difference was found between the two-level and three-level models it is not likely that our meta-analysis results were affected by dependent effect sizes' correlations.

Due to the difference in TMS timings of the included studies, we performed two metaregressions to test whether the stimulation timing could predict the effect size. The first metaregression was conducted using the TMS timing after stimulus offset for each study as a predictor variable, and indicated that TMS timing after stimulus offset does not predict the effect size, $Q M(1)=0.58, p=0.45$, indicating that TMS effects were likely similar between the included studies, irrelevant of when TMS was induced (Figure 5A). Further, since stimulus presentation differed between the included studies, we have performed a second meta-regression using TMS timing after stimulus onset as a predictor variable. Similarly, TMS timing after stimulus onset failed to predict the effect size $Q M(1)=0.49, \mathrm{p}=0.49$, indicating a similar TMS effect across the different TMS timing conditions (Figure 5B). A comparison of the two meta-regressions provided evidence for a very strong correlation $(\rho=$ $.98, p<.001)$, thus further supporting that the TMS effect is independent from the timing that TMS was induced (Figure 5C).

Facilitation versus inhibition. A third, exploratory, meta-analysis was conducted to explore whether there is evidence in favor of facilitatory or inhibitory TMS effects on VSTM performance. This meta-analysis was conducted on the signed effect sizes. Prior to the analysis the signs of effect sizes from studies measuring guess rates (van Lamsweerde et al., 2017), proportion of guesses (Koivisto et al., 2017), and absolute errors (Rademaker et al., 2017) were reversed, in order to indicate the same direction of effect as those measuring percent correct and detection sensitivity $\left(A^{\prime}\right)$. As such, positive values indicate facilitatory TMS effects on VSTM performance, while negative values represent inhibitory TMS effects.

For visualization purposes, studies were categorized into four groups (Figure 6), according to TMS timing (encoding or maintenance) and to the direction of the effect (inhibition or facilitation). Regarding encoding (Figure 6A), the random effects model provided evidence in favor of a moderate inhibition effect with an overall effect of $g=-.60$, $95 \% C I=-1.14,-.05, Z=-2.15, p=.031$. Contrary, there was evidence in favor of a small facilitation effect for maintenance (Figure $6 \mathrm{~B}$ ), $g=.32,95 \% C I=.09, .56, Z=2.67, p=.007$. 
Lastly, by considering all 32 effect sizes, there was no indication of a directional effect for sensory visual cortex TMS on VSTM performance (Figure 6C), since the overall random effects model failed to reach significance, $g=-.16,95 \% C I=-.49, .17, Z=-.95, p=.342$.

Overall, the exploratory meta-analysis indicates that the direction of TMS effects on VSTM performance differs between encoding and maintenance. In line with the well-studied role of the sensory visual cortex during VSTM encoding (e.g., Awh \& Jonides, 2001; D’Esposito \& Postle, 2015; de Graaf et al., 2014; Kammer, 2007; Masse et al., 2020; Serences, 2016; Shevlin, 2020), an inhibition effect (VSTM performance decrease) was evident when TMS was applied during the encoding VSTM phase. In contrast, a facilitation effect (VSTM performance increase) has been found for studies applying TMS during the maintenance VSTM phase. However, as also discussed below (see Discussion), TMS effects have been shown to be complex, both on the physiological and on the behavioral outcomes, and often depend on the specific stimulation parameters used, such as intensity, duration, and frequency (Aydin -Abidin et al., 2006; Eldaeif et al., 2011; Kammer et al., 2005; Moliadze et al., 2003). Further, as discussed above (see Data Analysis), in some cases the interpretation of the TMS effects on behavioral outcomes might be misinterpreted (e.g., when comparing the ipsilateral to the contralateral condition). Therefore, it is possible that different TMS parameters or experimental methods, lead to different behavioral effects. Nevertheless, the exploratory meta-analysis, which can be considered a more conservative approach since it is limited on quantifying the overall effect size of TMS beyond the direction of effects, reflects similar results as the encoding (see Meta-analysis 1: TMS interference during encoding) and maintenance (see Meta-analysis 2: TMS interference during maintenance) meta-analyses, by further confirming a stronger TMS effect during VSTM encoding and a weaker TMS effect during VSTM maintenance.

\section{Discussion}

A systematic review, two meta-analyses, two meta-regressions and one exploratory meta-analysis were conducted to investigate whether the sensory visual cortex is part of the brain network responsible for the encoding as well as the short-term maintenance of visual information. We focused on studies that interfered with sensory visual cortex using TMS during the encoding and maintenance phases of VSTM. The systematic review identified 14 papers that included 18 experiments and totaling 248 participants. Two meta-analyses were performed using the subset of studies that provided sufficient data for behavioral measure 
scores for a TMS and a control condition. The meta-analyses investigated separately the role of the sensory visual cortex in encoding and short-term maintenance of visual information. The meta-regressions explored whether the different stimulation timings of all included studies are related to the effect of the stimulation. The exploratory meta-analysis investigated the direction of the stimulation effects, indicating an inhibition effect for TMS during VSTM encoding and a facilitatory effect for TMS during VSTM maintenance. The findings indicate that encoding and maintaining visual information in VSTM are both similarly supported by a brain network that includes sensory visual cortex.

Our findings confirm the causal contribution of the sensory visual cortex in encoding of visual information in VSTM. In the six experiments described in the systematic review, all but one provided significant evidence that TMS during the encoding phase of VSTM can affect memory performance (Cattaneo et al., 2009; Koivisto et al., 2017; Rademaker et al., 2017; van Lamsweerde, \& Johnson, 2017). In addition, the quantitative analysis of the studies included in the meta-analysis further supports the presence of an effect of TMS on sensory visual cortex during the encoding phase of VSTM.

Previous neuroscientific evidence produced with various methodological approaches (e.g., Bettencourt, \& Xu, 2016; Lee et al., 2016; Lu et al., 2018; Tcheslavski et al., 2018) together with the findings of the present systematic review and the encoding meta-analysis, establish the involvement of the sensory visual cortex in encoding of visual information in VSTM. Here, we extend this previous evidence to now include evidence from studies that employed TMS. Moreover, replicating the well-established finding of the causal involvement of sensory visual cortex in encoding of visual information in VSTM provides further evidence for the validity of TMS in indeed being a suitable method to provide causal evidence for the neural activity subserving cognitive processing involved in encoding as well as maintenance of visual information in VSTM, as we discuss next.

The majority of the experiments reviewed here focused on the controversial role of the sensory visual cortex in the maintenance of visual information. Most of the TMS studies reviewed here investigating this question reported evidence supporting the sensory recruitment hypothesis (Cattaneo, et al., 2012; Rademaker et al., 2017; Saad et al., 2015; Silvanto \& Cattaneo, 2010; Silvanto, \& Soto, 2012; van de Ven et al., 2012; Zokaei et al., 2014). In addition, the findings of the meta-analysis of the available data coming from studies inducing TMS on the sensory visual cortex during the maintenance phase of the VSTM process, further support the sensory recruitment hypothesis by showing a significant effect of TMS. However, four experiments described in three different papers reported no significant 
effects of TMS, presenting evidence against the sensory recruitment hypothesis (Malik et al., 2015; Saad, \& Silvanto, 2013; Soto \& Silvanto, 2012). As previously discussed, even though three of these experiments failed to find direct TMS effects on memory performance, they reported an interaction between perception and memory processes which was evident by TMS interference. Such an interaction between perception and VSTM, indicates a shared neural substrate between the two mechanisms, which is consistent with predictions by the sensory recruitment hypothesis and our main findings (Pasternak, \& Greenlee, 2005; see also Lorenc, et al., 2021; Teng \& Postle, 2021).

Overall, the present systematic review and meta-analyses point to the direction of an involvement of the visual sensory cortex, not merely in the encoding, but also in the maintenance of visual information (Pasternak \& Greenlee, 2004). Our findings are not in full agreement with recent reviews, which suggest that there is insufficient evidence to support the sensory recruitment hypothesis (Xu, 2017, 2020, 2021). These reviews were heavily reliant on neuroimaging studies that are not suitable for detecting activity silent mechanisms thus ignoring any potential involvement of sensory visual cortex during memory maintenance (Masse et al., 2020; Oberauer, 2019; Rose et al., 2016; Serences, 2016; Sreenivasen et al., 2014). Considering recent evidence indicating that information in VSTM can be stored through synaptic weight changes and other activity-silent processes (Iamshchinina et al., 2021; Lorenc et al., 2018; Rose et al., 2016; Stokes, 2015; Zhang et al, 2021), it has been postulated that contemporary research should incorporate activity-silent mechanisms for studying VSTM, which in turn can reaffirm the role of the sensory visual cortex during VSTM maintenance (Lorenc \& Sreenivasan, 2021; Masse et al., 2020; Teng \& Postle, 2021; see also Adam et al., 2021; Beukers et al., 2021). Additionally, the activity-silent processes of the sensory visual cortex have been generating a lot of still unanswered questions, such as the one raised by Oberauer (2019) about whether neurally active representations are actually functionally important for maintaining information in working memory. To address these issues, we focused on studies using TMS for disrupting content-specific neural activity, thus providing causal evidence on the cognitive process subserved by activity of the brain area being targeted by TMS (de Graaf et al., 2014; Pitcher, et al., 2021; Sadrini, et al., 2011; Tapia \& Beck, 2014; van de Ven \& Sack, 2013). An additional explanation of the different conclusions between our study and those previous reports is the fact that many of the experiments identified here that showed an interference of TMS with the sensory visual cortex during the VSTM maintenance phase were not included in those reviews. This omission on behalf of the previous reviews showcases the importance of systematically 
searching the literature by following established guidelines (e.g., Moher et al., 2009, Page et al., 2021).

Our findings oppose previous reports (e.g., Xu, 2017, 2018, 2020, 2021), which suggest that any possible involvement of the sensory visual cortex during short-term maintenance is most likely a result of feedback from higher brain areas, such as the prefrontal and posterior parietal cortex. This suggestion was based on neuroimaging data showing that VSTM representations in the sensory visual cortex were wiped out at no behavioral cost, after task-irrelevant distractors were presented in a delayed estimation task (Bettencourt \& Xu, 2016; but see Rademaker et al., 2019 for a different result that is in line with the findings reported here). Similar to Betterncourt and Xu's (2016) findings, brain single-unit activity measurements in non-human primates support the idea that activity in the sensory visual cortex during VSTM maintenance likely reflect feedback from higher order areas (MendozaHalliday et al., 2014). However, considering the flexibility of the working memory system, where information can be transferred through interactions between sensory and frontal areas (Christophel et al., 2017; D’Esposito \& Postle, 2015; Teng \& Postle, 2021), this argument remains compatible with the idea that the sensory cortex is a necessary component of the network that underlies short-term maintenance of visual information (Gayet et al., 2018; Scimeca et al., 2018). These interactions are vital for memory maintenance in the sensory visual cortex and for other attentional processes (D'Esposito, 2007; D'Esposito \& Postle, 2015), meaning that activity in the frontal brain areas does not exclude or makes redundant the involvement of the sensory visual cortex during memory maintenance but rather highlights that the successful short-term maintenance of visual information relies on a network of brain areas instead of activity in isolated brain areas. The view of VSTM maintenance relying on a brain network, is in line with recent suggestions, which encourage future work to focus on how brain areas interact in this brain network during VSTM maintenance (Lorenc, \& Sreenivasan, 2021; Teng \& Postle, 2021).

An alternative explanation for the effects of TMS on memory performance reported here is that TMS does not interfere directly with the maintenance processes, but these effects reflect instead an interruption of attentional processes. Similar alternatives were proposed by previous reviews $(\mathrm{Xu}, 2017,2018,2020,2021)$, suggesting that the involvement of the sensory visual cortex might in fact echo feedback processing activity by higher-order brain areas (Miller et al., 1996) or deeper layers of the sensory visual cortex (Van Kerkoerle et al., 2017). For example, research suggests that sensory cortices are mediated by attentional mechanisms that synchronize neural oscillations (Bauer et al., 2020). Recently, it was shown 
that TMS induced in different frequencies can affect VSTM performance accordingly (Riddle et al., 2020). Yet, recent research provides evidence that working memory seems to similarly rely on phase-dependent oscillations (ten Oever et al., 2020). Future research on phasedependent cognitive mechanisms could possibly provide explanations relevant to the sensory recruitment hypothesis. For instance, future work could unveil if attention and working memory depend on the same oscillation phase (e.g., Arnulfo et al., 2020; Li et al., 2020) or if different frequency patterns explain each mechanism.

A limitation of the current study is the heterogeneity between the identified studies. This heterogeneity was expected, given the different methodological approaches, especially regarding the different parameters of TMS stimulation (de Graaf, \& Sack, 2011; Pitcher et al., 2021; Sadrini et al., 2011; van de Ven \& Sack, 2013). For example, even though a difference in the direction of effects was found, indicating inhibitory TMS effects during VSTM encoding compared to the facilitatory TMS effects during VSTM maintenance, this heterogeneity between the studies limits the conclusions that can be drawn by such findings. Specifically, because TMS effects on physiology and behavior can be complex (see below), it currently remains impossible to infer on the specific parameters driving the differences in the direction of stimulation effects. Ideally, a meta-analysis that groups each effect size according to its specific stimulation protocol and behavioral paradigm would provide important information regarding how sensory visual cortex TMS interferes with VSTM performance, but unfortunately, the small number of identified studies restrict us from such analyses. It should be noted, however, that because of the small number of the identified studies, the heterogeneity tests are rather indicative, and no strong conclusions can be drawn. Yet, the significant heterogeneity in the TMS studies exploring VSTM, raises some important issues that ought to be discussed and addressed. In view of the live debate around the sensory recruitment hypothesis (Ester et al., 2016; Gayet et al., 2018; Scimeca, et al., 2018; Shevlin, 2020; Teng, \& Postle, 2021; Xu, 2018), we think it is fundamental to focus on reproducible practices. Specifically, future studies should focus on specific methodological and technical approaches in such a manner that between study comparisons, both qualitatively and quantitatively, can be more accurately implemented (see Hardy, \& Thompson, 1998; Higgins, \& Thompson, 2002; Pitcher et al., 2021). Even though such heterogeneity in methods can be viewed as supporting our conclusions (i.e., the fact that TMS produced consistent effects across a different range of protocols speaks to the generalizability of the effects), future TMS research could benefit from focusing on more reproducible and open practices. For example, future studies should aim to report all relevant results, given that even null results in TMS 
studies are often informative and important (de Graaf, \& Sack, 2011). One way of promoting this is by preregistering experiments (see Nosek et al., 2018) and by uploading the raw data sets in open repositories, such as osf.io. It is also suggested that future studies offer sufficient information regarding TMS parameters (e.g., localization, power output, coil position, frequency) in a manner which can guide and promote reproducibility (see Peterchev et al., 2012). Further, TMS studies in the field of cognitive neuroscience should carefully design their experiments (e.g., use more than one control condition) in order to produce more reliable results (Pitcher et al., 2021; Sadrini et al., 2011).

Another important issue that needs to be raised is the dependency of the calculated effect sizes. Given that some individuals participated in more than one experiment and since in some cases more than one effect size was calculated for some of the included studies in the meta-analyses, it is possible that some effect sizes are biased, since they are not independent (Cheung, 2019). Nevertheless, because of the small number of available datasets we proceeded with the most conservative approach to our meta-analyses, namely the random effect model. In order to explore if the dependency of some of the data could account for our results, we clustered each experimental condition to its corresponding study, thus creating an additional level in each of our meta-analysis models. This additional study level could not explain the variance in our meta-analyses models indicating that the results were likely not affected by correlations in the dependent effect sizes.

Significant small study bias was identified, which was reflected in the asymmetry of the funnel plot and the significant statistical test for small study bias in the encoding metaanalysis. This could indicate a possible publication bias, often referred to as the file drawer problem (e.g., Nagarajan et al., 2017; see also Friese, \& Frankenbach, 2019), which has been shown to be common in cognitive neuroscience (Huber et al., 2019). However, because of the small number of identified studies and the fact that almost half of these studies explored a different primary question to the one explored through our meta-analyses, no robust conclusions regarding this kind of bias can be drawn. However, it must be noted that publication bias can indeed affect the results of the meta-analysis both for the $Q$ test, as well as the heterogeneity tests by increasing or decreasing the value of the true effect sizes (Augusteijn et al., 2019; Friese, \& Frankenbach, 2019). In addition, half of the identified studies did not provide sufficient statistical data in their published work in order to be included in the meta-analyses. This limitation, of the current literature, combined with the file drawer problem, causes a drawback for meta-science and confines reproducible science. We strongly suggest that researchers and publishers, should aim to rigorously present all relevant 
data in their publications in order to address this concern. In general, to reduce bias scientists and journals should be encouraged to publish with a focus on robust scientific methodology as opposed to whether results are significant or not.

Another important factor that needs to be considered when interpreting these studies is the complexity of the TMS effects, both physiologically, and then on the observable behavioral output. TMS can indeed help infer causal relationships between brain activity and behavior (Bergmann, \& Hartwigsen, 2021; de Graaf, \& Sack, 2011; Hallett, 2000; PascualLeone et al., 2000; Pitcher et al., 2020; Sack, 2006; Sandrini et al., 2011; Siebner et al., 2009). However, the effects of TMS stimulation are indeed complex, both on the physiology and on the observed behavioral measure (Bergmann, \& Hartwigsen, 2021; de Graaf, \& Sack, 2011; Harris et al., 2008; Pitcher et al., 2020; Silvanto \& Cattaneo, 2017) and as such any effects need to be interpreted with caution. For example, both the "virtual lesion" and "neural noise" descriptions have been criticized as too simplistic and thus inadequate to describe the true complexity of the TMS effects (Bergmann, \& Hartwigsen, 2021; Harris et al., 2008; Silvanto \& Cattaneo, 2017), as TMS has been shown to interfere with both feedforward and feedback processes (Kim \& Freeman, 2014), with activity silent mechanisms (Rose et al., 2016), with oscillatory activity (Riddle et al., 2020), between sub-cortical and intra-cortical inputs (Kim et al., 2015), and between brain regions (Ruff et al., 2008). Physiologically, TMS on the sensory visual cortex was shown to result in highly variable effects described as facilitatory or suppressive (or both) for neural activity, depending on various factors. Some of these factors include stimulation intensity (Kammer et al., 2005), stimulation duration and frequency (Aydin -Abidin et al., 2006; Eldaeif et al., 2011; Moliadze et al., 2003), eyemovements (Silva et al., 2021), and tuning properties (i.e., stimulus orientation, contrast, spatial frequency; Kim et al., 2015). Further, evidence from single-unit recordings indicated that TMS effects are state-dependent, such that greater TMS effects are expected when neural activity is higher before stimulation (Pasley et al., 2009). Similarly, these state-dependent effects have been reflected in behavioral outcomes, for example when specific visual stimuli are primed (e.g., congruent vs. incongruent primer; Silvanto et al., 2018) or when spatial attention is required (e.g., attended vs. unattended locations; Bestmann et al., 2007). The complexity of the stimulation effects using TMS should therefore be taken into consideration when interpreting TMS studies reporting TMS effects (or lack of effects), especially when inferring the neural mechanism of such effects. For example, cognition is often viewed through a strictly modular approach, where the neural activity in a specific ROI is considered responsible for the observed behavior (e.g., the 'Sheringtonian' view; Barack \& Krakauer, 
2021). This may lead to strictly modular TMS cause-and-effect relationships between a particular ROI and an observed behavior, and consequently the neural activity in the ROI is viewed as either the cause or not, of the observed behavior. As described above, the complexity of TMS effects restricts such modular cause-and-effect inferences. Nonetheless, it is reasonable to assume that the TMS effects on the observed behavior provide causal information regarding the brain network that underlies the cognitive process under investigation (Bergmann \& Hartwigsen, 2021; Pitcher et al., 2020).

Considering the complexity of TMS effects on the brain, an alternative explanation of the effects of sensory visual cortex TMS on VSTM performance suggests that TMS interferes with downstream processes of higher order brain areas such as the posterior parietal cortex (e.g., Xu, 2017), and not with maintenance of visual information in sensory visual cortex per se. However, we think it is unlikely that our meta-analytic effects reflect such downstream effects of VSTM performance. Specifically, it has been systematically reported that to be able to limit inferences within a specific brain network, multiple control TMS conditions need to be considered (Bergmann \& Hartwigsen, 2021; Duecker \& Sack, 2015; Pitcher et al., 2021). The current meta-analyses include effect sizes from numerous control conditions (see Table 2 and Table 3), which collectively lead to the conclusion that sensory visual cortex TMS significantly affects VSTM performance, thus supporting the idea that differences on behavioral outcomes can likely be attributed to interference with sensory visual cortex processing. Moreover, the comparison of the two meta-analyses and the two meta-regressions further supports the idea that behavioral differences are not epiphenomenal but rather due to processes in the sensory visual cortex. Specifically, the meta-analysis comparisons and the meta-regressions indicate that TMS effects during VSTM maintenance are similar to the effects during encoding, which are expected due to the established role of sensory visual cortex in encoding (Awh \& Jonides, 2001; D'Esposito, \& Postle, 2015; de Graaf et al., 2014; Kammer, 2007; Masse et al., 2020; Serences, 2016; Shevlin, 2020; Xu, 2017, 2020, 2021). Despite the above, because the current study focuses only on studies interfering with TMS on the sensory visual cortex, we cannot completely rule out the possibility that such TMS behavioral effects are due to interference with downstream processes of higher brain areas such as the posterior parietal cortex. Future work could address this issue by directly comparing TMS effects on sensory visual cortex versus TMS on higher brain areas such as parietal cortex (e.g., Prime et al., 2008) or prefrontal cortex (e.g., Lorenc et al., 2015; see also Panichello \& Buschman, 2021) during VSTM maintenance. As such, to improve our understanding of the underlying brain network involved in the maintenance of information 
during VSTM, future work should move beyond the modular view of focusing on the contribution of a single brain area and towards the study of brain networks and functional connectivity of brain areas involved in VSTM (for similar arguments see Lorenc \& Sreenivasan, 2021; Teng \& Postle, 2021).

Beyond these limitations, the current systematic review and meta-analyses were informative in several ways. As formerly discussed, previous reviews on the topic (Xu, 2017, 2018,2020 ) failed to mention some of the relevant studies, which were likely missed because the identification did not follow a systematic approach (e.g., Moher et al., 2009; Page et al., 2021). Further, the meta-analyses allowed us to quantify the effect size of TMS over the sensory visual cortex on VSTM performance, indicating a high and a moderate effect for encoding and maintenance, respectively. Finally, our systematic review and meta-analyses provide a summary of the current state of the art on TMS literature exploring the role of the sensory visual cortex in VSTM. This summary, further to the support of the sensory visual cortex involvement in VSTM maintenance, signifies some of the limitations of the current TMS literature, thus signaling drawbacks that future work can focus on and address. Specifically, future work could aim to explore the contradictory effects reported between the studies, by investigating the role of the sensory visual cortex in VSTM, while presenting stimuli monocularly (e.g., Carmel et al., 2010), or by testing how TMS interferes with different storage processes (Lorenc et al., 2020; see also Beukers et al., 2021). Also, forthcoming experiments studying the role of the sensory visual cortex should carefully design their stimuli, so that they correspond to elemental features processed by sensory areas (Harrison \& Tong, 2009; Kim et al., 2015; Serences et al., 2009), and consider the possible interaction of memory and perceptual load (Konstantinou, et al., 2012, 2014; Konstantinou, \& Lavie, 2013).

In summary, evidence from studies that interfered with sensory visual cortex activity using TMS during VSTM, support the involvement of the sensory visual cortex in VSTM encoding as well as VSTM maintenance. Interestingly, TMS in low VSTM load conditions is not as effective as with higher load, and similarly, increased perceptual demands can modulate TMS effects. Given the importance of reproducible practices, it is suggested that the specific parameters of stimulation using TMS are carefully implemented, which will encourage future and more robust systematic and meta-analytic approaches to cognitive science. 


\section{Conclusion}

The causal evidence that was systematically reviewed here, derived from the TMS studies investigating the role of the sensory visual cortex in VSTM, seem to be support our hypothesis that sensory visual cortex is a necessary component of the brain network that underlies both the encoding as well as the short-term maintenance of visual information, in line with the sensory recruitment hypothesis (Awh \& Jonides, 2001; Christophel et al., 2017; Lorenc et al., 2021; Pasternak, \& Greenlee, 2005; Serences, 2016; Sreenivasen et al., 2014; Teng \& Postle, 2021). Further to the well-established involvement of the sensory visual cortex during the encoding of visual information in VSTM, results from numerous TMS experiments indicate that the role of the sensory visual cortex goes beyond the initial encoding and consolidation phases and is also involved in the maintenance of memory representations. Even though some studies failed to detect a TMS effect, an interaction between perception and VSTM was evident, a finding that supports a possible shared neural mechanism between perception and VSTM in the sensory visual cortex. Quantifying the results of the available data using meta-analytic methodology, further supports that the sensory visual cortex is indeed involved in encoding as well as short-term memory maintenance by favoring the TMS condition over the control condition as indicated by behavioral outcomes. 


\section{References}

References marked with the symbol " $\wedge$ " were included in the systematic review.

References marked with an asterisk (*) indicate studies that were further included in the meta-analysis.

Adam, K. C. S., Rademaker, R. L., \& Serences, J. (2021, June 15). Evidence for, and challenges to, sensory recruitment models of visual working memory. PsyArxiv [Accessed 24 November 2021] https://doi.org/10.31234/osf.io/wb5e6

Arnulfo, G., Wang, S. H., Myrov, V., Toselli, B., Hirvonen, J., Fato, M. M., ... \& Palva, S. (2020). Long-range phase synchronization of high-frequency oscillations in human cortex. Nature communications, 11(1), 1-15. https://doi.org/10.1038/s41467-020-18975$\underline{8}$

Assink, M., \& Wibbelink, C. J. (2016). Fitting three-level meta-analytic models in R: A stepby-step tutorial. The Quantitative Methods for Psychology, 12(3), 154-174. https://doi.org/10.20982/tqmp.12.3.p154

Augusteijn, H. E., van Aert, R., \& van Assen, M. A. (2019). The effect of publication bias on the Q test and assessment of heterogeneity. Psychological methods, 24(1), 116. https://doi.org/10.1037/met0000197

Awh, E., \& Jonides, J. (2001). Overlapping mechanisms of attention and spatial working memory. Trends in cognitive sciences, 5(3), 119-126. https://doi.org/10.1016/s13646613(00)01593-x

Aydin-Abidin, S., Moliadze, V., Eysel, U. T., \& Funke, K. (2006). Effects of repetitive TMS on visually evoked potentials and EEG in the anaesthetized cat: dependence on stimulus frequency and train duration. The Journal of physiology, 574(Pt 2), 443-455. https://doi.org/10.1113/jphysiol.2006.108464

Bauer, A. K. R., Debener, S., \& Nobre, A. C. (2020). Synchronisation of Neural Oscillations and Cross-modal Influences. Trends in Cognitive Sciences. https://doi.org/10.1016/j.tics.2020.03.003

Bays, P. M., Gorgoraptis, N., Wee, N., Marshall, L., \& Husain, M. (2011). Temporal dynamics of encoding, storage, and reallocation of visual working memory. Journal of vision, 11(10), 6-6. https://doi.org/10.1167/11.10.6

Barack, D. L., \& Krakauer, J. W. (2021). Two views on the cognitive brain. Nature Reviews Neuroscience, 22(6), 359-371. https://doi.org/10.1038/s41583-021-00448-6

Bergmann, T. O., \& Hartwigsen, G. (2021). Inferring causality from noninvasive brain stimulation in cognitive neuroscience. Journal of cognitive neuroscience, 33(2), $195-$ 225. https://doi.org/10.1162/jocn_a 01591

Bettencourt, K. C., \& Xu, Y. (2016). Decoding the content of visual short-term memory under distraction in occipital and parietal areas. Nature neuroscience, 19(1), 150. https://doi.org/10.1038/nn.4174

Bestmann, S., Ruff, C. C., Blakemore, C., Driver, J., \& Thilo, K. V. (2007). Spatial attention changes excitability of human visual cortex to direct stimulation. Current Biology, 17(2), 134-139. https://doi.org/10.1016/j.cub.2006.11.063

Beukers, A. O., Buschman, T. J., Cohen, J. D., \& Norman, K. A. (2021). Is Activity Silent Working Memory Simply Episodic Memory?. Trends in Cognitive Sciences. https://doi.org/10.1016/j.tics.2021.01.003 
Bisley, J. W., \& Pasternak, T. (2000). The multiple roles of visual cortical areas MT/MST in remembering the direction of visual motion. Cerebral Cortex, 10(11), 1053-1065. https://doi.org/10.1093/cercor/10.11.1053

Brady, T. F., Störmer, V. S., \& Alvarez, G. A. (2016). Working memory is not fixedcapacity: More active storage capacity for real-world objects than for simple stimuli. Proceedings of the National Academy of Sciences, 113(27), 7459-7464. https://doi.org/10.1073/pnas.1520027113

Brockmole, J. R., Wang, R. F., \& Irwin, D. E. (2002). Temporal integration between visual images and visual percepts. Journal of Experimental Psychology: Human Perception and Performance, 28(2), 315-334. https://doi.org/10.1037/0096-1523.28.2.315

Carmel, D., Arcaro, M., Kastner, S., \& Hasson, U. (2010). How to create and use binocular rivalry. JoVE (Journal of Visualized Experiments), (45), e2030. https://doi.org/10.3791/2030

${ }^{\wedge}$ Cattaneo, Z., Bona, S., \& Silvan to, J. (2012). Cross-adaptation combined with TMS reveals a functional overlap between vision and imagery in the early visual cortex. NeuroImage, 59(3), 3015-3020. https://doi.org/10.1016/j.neuroimage.2011.10.022

*Cattaneo, Z., Vecchi, T., Pascual-Leone, A., \& Silvanto, J. (2009). Contrasting early visual cortical activation states causally involved in visual imagery and short-term memory. The European Journal of Neuroscience, 30(7), 1393-1400. https://doi.org/10.1111/j.1460-9568.2009.06911.x

Chafee, M. V., \& Goldman-Rakic, P. S. (1998). Matching patterns of activity in primate prefrontal area $8 \mathrm{a}$ and parietal area 7ip neurons during a spatial working memorytask. Journal of neurophysiology, 79(6), 2919-2940. https://doi.org/10.1152/jn.1998.79.6.2919

Cheung, M. W. L. (2014). Modeling dependent effect sizes with three-level meta-analyses: a structural equation modeling approach. Psychological Methods, 19(2), 211. https://doi.org/10.1037/a0032968

Cheung, M. W. L. (2019). A guide to conducting a meta-analysis with non-independent effect sizes. Neuropsychology review, 1-10. https://doi.org/10.1007/s11065-019-09415$\underline{6}$

Christophel, T. B., Allefeld, C., Endisch, C., \& Haynes, J. D. (2018). View-independent working memory representations of artificial shapes in prefrontal and posterior regions of the human brain. Cerebral Cortex, 28(6), 2146-2161. https://doi.org/10.1093/cercor/bhx119

Christophel, T. B., Klink, P. C., Spitzer, B., Roelfsema, P. R., \& Haynes, J. D. (2017). The distributed nature of working memory. Trends in Cognitive Sciences, 21(2), 111-124. https://doi.org/10.1016/j.tics.2016.12.007

Cowan, N., Elliott, E. M., Saults, J. S., Morey, C. C., Mattox, S., Hismjatullina, A., \& Conway, A. R. (2005). On the capacity of attention: Its estimation and its role in working memory and cognitive aptitudes. Cognitive Psychology, 51, 42100. https://doi.org/10.1016/j.cogpsych.2004.12.001

Clegg, B. A., DiGirolamo, G. J., \& Keele, S. W. (1998). Sequence learning. Trends in cognitive sciences, 2(8), 275-281. https://doi.org/10.1016/S1364-6613(98)01202-9

Curtis, C. E., \& D'Esposito, M. (2003). Persistent activity in the prefrontal cortex during working memory. Trends in cognitive sciences, 7(9), 415-423. https://doi.org/10.1016/S1364-6613(03)00197-9 
de Graaf, T. A., \& Sack, A. T. (2011). Null results in TMS: from absence of evidence to evidence of absence. Neuroscience \& Biobehavioral Reviews, 35(3), 871-877. https://doi.org/10.1016/j.neubiorev.2010.10.006

de Graaf, T. A., Koivisto, M., Jacobs, C., \& Sack, A. T. (2014). The chronometry of visual perception: review of occipital TMS masking studies. Neuroscience \& Biobehavioral Reviews, 45, 295-304. https://doi.org/10.1016/j.neubiorev.2014.06.017

D'Esposito, M. (2007). From cognitive to neural models of working memory. Philosophical Transactions of the Royal Society B: Biological Sciences, 362(1481), 761-772. https://doi.org/10.1098/rstb.2007.2086

D'Esposito, M., \& Postle, B. R. (2015). The cognitive neuroscience of working memory. Annual review of psychology, 66, 115-142. https://doi.org/10.1146/annurevpsych-010814-015031

D'Esposito, M., Zarahn, E., \& Aquirre, G. K. (1999). Event-related functional MRI: implications for cognitive psychology. Psychological bulletin, 125(1), 155-164. https://doi.org/10.1037/0033-2909.125.1.155

Duecker, F., \& Sack, A. T. (2015). Rethinking the role of sham TMS. Frontiers in psychology, 6, 210. https://doi.org/10.3389/fpsyg.2015.00210

Di Lollo, V., \& Dixon, P. (1988). Two forms of persistence in visual information processing. Journal of Experimental Psychology: Human Perception and Performance, 14(4), 671-681. https://doi.org/10.1037/0096-1523.14.4.671

Egger, M., Smith, G.D., Schneider, M., \& Minder, C., 1997. Bias in meta-analysis detected by a simple, graphical test. Bmj, 315(7109), 629-634. https://doi.org/10.1136/bmj.315.7109.629

Eldaief, M. C., Halko, M. A., Buckner, R. L., \& Pascual-Leone, A. (2011). Transcranial magnetic stimulation modulates the brain's intrinsic activity in a frequency-dependent manner. Proceedings of the National Academy of Sciences of the United States of America, 108(52), 21229-21234. https://doi.org/10.1073/pnas.1113103109

Ester, E. F., Rademaker, R. L., \& Sprague, T. C. (2016). How do visual and parietal cortex contribute to visual short-term memory?. ENeuro, 3(2). https://doi.org/10.1523/eneuro.0041-16.2016

Ester, E. F., Sprague, T. C., \& Serences, J. T. (2015). Parietal and frontal cortex encode stimulus-specific mnemonic representations during visual working memory. Neuron, 87(4), 893-905. https://doi.org/10.1016/j.neuron.2015.07.013

Funahashi, S. (2017). Working memory in the prefrontal cortex. Brain sciences, 7(5), 49. https://doi.org/10.1016/j.neuron.2015.07.013

Fleiss, J., L., (1993). "Review Papers: The Statistical Basis of Meta-Analysis." Statistical Methods in Medical Research 2 (2). Sage Publications Sage CA: Thousand Oaks, CA: 121-45. https://doi.org/10.1177/096228029300200202

Friese, M., \& Frankenbach, J. (2019). p-Hacking and publication bias interact to distort metaanalytic effect size estimates. Psychological Methods. https://doi.org/10.1037/met0000246

Fritz, C. O., Morris, P. E., \& Richler, J. J. (2012). Effect size estimates: current use, calculations, and interpretation. Journal of experimental psychology: General, 141(1), 2. https://doi.apa.org/doi/10.1037/a0024338

Funahashi, S., Bruce, C. J., \& Goldman-Rakic, P. S. (1989). Mnemonic coding of visual space in the monkey's dorsolateral prefrontal cortex. Journal of neurophysiology, 61(2), 331-349. https://doi.org/10.1152/jn.1989.61.2.331 
Gayet, S., Paffen, C. L., \& Van der Stigchel, S. (2013). Information matching the content of visual working memory is prioritized for conscious access. Psychological Science, 24(12), 2472-2480. https://doi.org/10.1177\%2F0956797613495882

Gayet, S., Paffen, C. L., \& Van der Stigchel, S. (2018). Visual working memory storage recruits sensory processing areas. Trends in cognitive sciences, 22(3), 189-190. https://doi.org/10.1016/j.tics.2017.09.011

Grange, J. A., Moore, S. B., \& Berry, E. D. (2021). mixtur: An R package for designing, analysing, and modelling continuous report visual short-term memory studies. https://doi.org/10.31234/osf.io/n6gqx [preprint]

Hallett, M. (2000). Transcranial magnetic stimulation and the human brain. Nature, 406(6792), 147-150. https://doi.org/10.1038/35018000

Hardy, R. J., \& Thompson, S. G. (1998). Detecting and describing heterogeneity in metaanalysis. Statistics in medicine, 17(8), 841-856. https://doi.org/10.1002/(sici)10970258(19980430)17:8\%3C841::aid-sim781\%3E3.0.co;2-d

Harrer, M., Cuijpers, P., Furukawa, T. A., \& Ebert, D. D. (2019). Doing meta-analysis in R: a hands-on guide. PROTECT Lab Erlangen. https://doi.org/10.5281/zenodo. 2551803

Harris, J. A., Clifford, C. W., \& Miniussi, C. (2008). The functional effect of transcranial magnetic stimulation: signal suppression or neural noise generation?. Journal of Cognitive Neuroscience, 20(4), 734-740. https://doi.org/10.1162/jocn.2008.20048

Harrison, S. A., \& Tong, F. (2009). Decoding reveals the contents of visual working memory in early visual areas. Nature, 458(7238), 632-635. https://doi.org/10.1038/nature07832

Hedges, L., V., (1981). “Distribution Theory for Glass's Estimator of Effect Size and Related Estimators.” Journal of Educational Statistics 6 (2). Sage Publications Sage CA:

Thousand Oaks, CA: 107-28. https://doi.org/10.2307/1164588

Higgins, J. P. T., Thompson, S. G., Deeks, J. J., \& Altman, D. G. (2003). Measuring inconsistency in meta-analyses. British Medical Journal, 327, 557-560. https://doi.org/10.1136/bmj.327.7414.557

Higgins, J. P., \& Thompson, S. G. (2002). Quantifying heterogeneity in a metaanalysis. Statistics in medicine, 21(11), 1539-1558. https://doi.org/10.1002/sim.1186

Hitch, G. J., Allen, R. J., \& Baddeley, A. D. (2020). Attention and binding in visual working memory: Two forms of attention and two kinds of buffer storage. Attention, Perception, \& Psychophysics, 82(1), 280-293. https://doi.org/10.3758/s13414-019-01837-x

Huber, D. E., Potter, K. W., \& Huszar, L. D. (2019). Less "Story" and more "Reliability" in cognitive neuroscience. Cortex; a journal devoted to the study of the nervous system and behavior, 113, 347. https://doi.org/10.1016/j.cortex.2018.10.030

Issa, N. P., Rosenberg, A., \& Husson, T. R. (2008). Models and measurements of functional maps in V1. Journal of neurophysiology, 99(6), 2745-2754. https://doi.org/10.1152/jn.90211.2008

*Jia, K., Li, Y., Gong, M., Huang, H., Wang, Y., \& Li, S. (2021). Perceptual learning beyond perception: mnemonic representation in early visual cortex and intraparietal sulcus. Journal of Neuroscience. https://doi.org/10.1523/JNEUROSCI.2780-20.2021

Jolicœur, P., \& Dell'Acqua, R. (1998). The demonstration of short-term consolidation. Cognitive psychology, 36(2), 138-202. https://doi.org/10.1006/cogp.1998.0684

Joukal, M. (2017). Anatomy of the human visual pathway. In Homonymous visual field defects (pp. 1-16). Springer, Cham. https://doi.org/10.1007/978-3-319-52284-5_1 
Kammer, T. (2007). Visual masking by transcranial magnetic stimulation in the first 80 milliseconds. Advances in Cognitive Psychology, 3(1-2), 177-179. https://doi.org/10.2478/v10053-008-0023-2

Kammer, T., Puls, K., Erb, M., \& Grodd, W. (2005). Transcranial magnetic stimulation in the visual system. II. Characterization of induced phosphenes and scotomas. Experimental brain research, 160(1), 129-140. https://doi.org/10.1007/s00221-004-1992-0

Kim, T., Allen, E. A., Pasley, B. N., \& Freeman, R. D. (2015). Transcranial magnetic stimulation changes response selectivity of neurons in the visual cortex. Brain stimulation, 8(3), 613-623. https://doi.org/10.1016/j.brs.2015.01.407

Kim, T., \& Freeman, R. D. (2014). Selective stimulation of neurons in visual cortex enables segregation of slow and fast connections. Neuroscience, 274, 170-186. https://doi.org/10.1016/j.neuroscience.2014.05.041

Kiyonaga, A., Scimeca, J. M., Bliss, D. P., \& Whitney, D. (2017). Serial dependence across perception, attention, and memory. Trends in Cognitive Sciences, 21(7), 493-497. https://doi.org/10.1016/j.tics.2017.04.011

Knight, R. T., Staines, W. R., Swick, D., \& Chao, L. L. (1999). Prefrontal cortex regulates inhibition and excitation in distributed neural networks. Acta psychologica, 101(2-3), 159-178. https://doi.org/10.1016/S0001-6918(99)00004-9

*Koivisto, M., Harjuniemi, I., Railo, H., Salminen-Vaparanta, N., \& Revonsuo, A. (2017). Transcranial magnetic stimulation of early visual cortex suppresses conscious representations in a dichotomous manner without gradually decreasing their precision. NeuroImage, 158, 308. https://doi.org/10.1016/j.neuroimage.2017.07.011

Konstantinou, N., \& Lavie, N. (2013). Dissociable roles of different types of working memory load in visual detection. Journal of Experimental Psychology: Human Perception and Performance, 39(4), 919. https://doi.org/10.1037/a0033037

Konstantinou, N., \& Lavie, N. (2020). Effects of visual short-term memory load and attentional demand on the contrast response function. Journal of Vision, 20(10), 6-6. https://doi.org/10.1167/jov.20.10.6

Konstantinou, N., Bahrami, B., Rees, G., \& Lavie, N. (2012). Visual short-term memory load reduces retinotopic cortex response to contrast. Journal of Cognitive Neuroscience, 24(11), 2199-2210. https://doi.org/10.1162/jocn_a 00279

Konstantinou, N., Beal, E., King, J. R., \& Lavie, N. (2014). Working memory load and distraction: dissociable effects of visual maintenance and cognitive control. Attention, Perception, \& Psychophysics, 76(7), 1985-1997. https://doi.org/10.3758/s13414-014$\underline{0742-\mathrm{Z}}$

Konstantinou, N., Constantinidou, F., \& Kanai, R. (2016). Discrete capacity limits and neuroanatomical correlates of visual short-term memory for objects and spatial locations. Human brain mapping, 38(2), 767-778. https://doi.org/10.1002/hbm.23416

Lamme, V. A., Super, H., Landman, R., Roelfsema, P. R., \& Spekreijse, H. (2000). The role of primary visual cortex (V1) in visual awareness. Vision research, 40(10-12), 15071521. https://doi.org/10.1016/S0042-6989(99)00243-6

Leavitt, M. L., Mendoza-Halliday, D., \& Martinez-Trujillo, J. C. (2017). Sustained Activity Encoding Working Memories: Not Fully Distributed. Trends in Neurosciences, 40(6), 328-346. https://doi.org/10.1016/j.tins.2017.04.004

Lee, S. H., Kravitz, D. J., \& Baker, C. I. (2013). Goal-dependent dissociation of visual and prefrontal cortices during working memory. Nature neuroscience, 16(8), 997. https://doi.org/10.1038/nn.3452 
Lee, W., Kim, H. C., Jung, Y., Chung, Y. A., Song, I. U., Lee, J. H., \& Yoo, S. S. (2016). Transcranial focused ultrasound stimulation of human primary visual cortex. Scientific reports, 6(1), 1-12. https://doi.org/10.1038/srep34026

Levy, R., \& Goldman-Rakic, P. S. (2000). Segregation of working memory functions within the dorsolateral prefrontal cortex. In Executive control and the frontal lobe: Current issues (pp. 23-32). Springer, Berlin, Heidelberg. https://doi.org/10.1007/978-3-64259794-7 4

Li, D., Zhao, C., Guo, J., Kong, Y., Li, H., Du, B., ... \& Song, Y. (2020). Visual Working Memory Guides Spatial Attention: Evidence from Alpha Oscillations and Sustained Potentials. Neuropsychologia, 107719.

https://doi.org/10.1016/j.neuropsychologia.2020.107719

Lorenc, E. S., Lee, T. G., Chen, A. J. W., \& D'Esposito, M. (2015). The effect of disruption of prefrontal cortical function with transcranial magnetic stimulation on visual working memory. Frontiers in systems neuroscience, 9, 169. https://doi.org/10.3389/fnsys.2015.00169

Lorenc, E. S., Sreenivasan, K. K., Nee, D. E., Vandenbroucke, A. R., \& D'Esposito, M. (2018). Flexible coding of visual working memory representations during distraction. Journal of Neuroscience, 38(23), 5267-5276. https://doi.org/10.1523/JNEUROSCI.3061-17.2018

Lorenc, E. S., Mallett, R., \& Lewis-Peacock, J. A. (2021). Distraction in Visual Working Memory: Resistance is Not Futile. Trends in Cognitive Sciences. https://doi.org/10.1016/j.tics.2020.12.004

Lorenc, E. S., \& Sreenivasan, K. K. (2021). Reframing the debate: The distributed systems view of working memory. Visual Cognition, 1-9. https://doi.org/10.1080/13506285.2021.1899091

Lu, Y., Yin, J., Chen, Z., Gong, H., Liu, Y., Qian, L., ... \& Wang, W. (2018). Revealing detail along the visual hierarchy: neural clustering preserves acuity from V1 to V4. Neuron, 98(2), 417-428. https://doi.org/10.1016/j.neuron.2018.04.003

Luck, S. J., \& Vogel, E. K. (1997). The capacity of visual working memory for features and conjunctions. Nature, 390, 279-281. https://doi.org/10.1038/36846

Lüdecke, D., (2018). Effect Size Computation for Meta Analysis. https://CRAN.Rproject.org/package $=$ esc.

Lundqvist, M., Herman, P., \& Miller, E. K. (2018). Working memory: delay activity, yes! Persistent activity? Maybe not. Journal of Neuroscience, 38(32), 7013-7019. https://doi.org/10.1523/JNEUROSCI.2485-17.2018

^Malik, P., Dessing, J. C., \& Crawford, J. D. (2015). Role of early visual cortex in transsaccadic memory of object features. Journal of Vision, 15(11), 1-17. https://doi.org/10.1167/15.11.7

Magnussen, S., Greenlee, M. W., Asplund, R., \& Dyrnes, S. (1991). Stimulus-specific mechanisms of visual short-term memory. Vision research, 31(7-8), 1213-1219. https://doi.org/10.1016/0042-6989(91)90046-8

Masse, N. Y., Rosen, M. C., \& Freedman, D. J. (2020). Reevaluating the Role of Persistent Neural Activity in Short-Term Memory. Trends in Cognitive Sciences, 24(3), 242258. https://doi.org/10.1016/j.tics.2019.12.014

McKeefry, D. J., Burton, M. P., \& Vakrou, C. (2007). Speed selectivity in visual short term memory for motion. Vision research, 47(18), 2418-2425. https://doi.org/10.1016/j.visres.2007.05.011 
Mendoza-Halliday, D., Torres, S., \& Martinez-Trujillo, J. C. (2014). Sharp emergence of feature-selective sustained activity along the dorsal visual pathway. Nature neuroscience, 17(9), 1255. https://doi.org/10.1038/nn.3785

Mikolajewicz, N., \& Komarova, S. V. (2019). Meta-analytic methodology for basic research: A practical guide. Frontiers in Physiology, 10. https://doi.org/10.3389/fphys.2019.00203

Miller, E. K., \& Cohen, J. D. (2001). An integrative theory of prefrontal cortex function. Annual review of neuroscience, 24(1), 167-202. https://doi.org/10.1146/annurev.neuro.24.1.167

Miller, E. K., Erickson, C. A., \& Desimone, R. (1996). Neural mechanisms of visual working memory in prefrontal cortex of the macaque. Journal of neuroscience, 16(16), 51545167. https://doi.org/10.1523/JNEUROSCI.16-16-05154.1996

Moher, D., Liberati, A., Tetzlaff, J., \& Altman, D. G. (2009). Preferred reporting items for systematic reviews and meta-analyses: the PRISMA statement. Annals of internal medicine, 151(4), 264-269. https://doi.org/10.1371/journal.pmed.1000097

Moliadze, V., Zhao, Y., Eysel, U., \& Funke, K. (2003). Effect of transcranial magnetic stimulation on single-unit activity in the cat primary visual cortex. The Journal of physiology, 553(2), 665-679. https://doi.org/10.1113/jphysiol.2003.050153

Morrissey, M. B. (2016). Meta-analysis of magnitudes, differences and variation in evolutionary parameters. Journal of Evolutionary Biology, 29(10), 1882-1904. https://doi.org/10.1111/jeb.12950

Nagarajan, P., Garla, B., Taranath, M., \& Nagarajan, I. (2017). The file drawer effect: A call for meticulous methodology and tolerance for non-significant results. Indian Journal of Anesthesia, 61(6). https://doi.org/10.4103/ija.ija_280_17

Nosek, B. A., Ebersole, C. R., DeHaven, A. C., \& Mellor, D. T. (2018). The preregistration revolution. Proceedings of the National Academy of Sciences, 115(11), 2600-2606. https://doi.org/10.1073/pnas.1708274114

Oberauer, K. (2019). Working Memory and Attention-A Conceptual Analysis and Review. Journal of cognition, 2(1). https://doi.org/10.5334/joc.58

Page, M. J., Moher, D., Bossuyt, P. M., Boutron, I., Hoffmann, T. C., Mulrow, C. D., ... \& McKenzie, J. E. (2021). PRISMA 2020 explanation and elaboration: updated guidance and exemplars for reporting systematic reviews. $b m j, 372$. https://doi.org/10.1136/bmj.n71

Panichello, M. F., \& Buschman, T. J. (2021). Shared mechanisms underlie the control of working memory and attention. Nature, 592(7855), 601-605. https://doi.org/10.1038/s41586-021-03390-w

Pascual-Leone, A., Walsh, V., \& Rothwell, J. (2000). Transcranial magnetic stimulation in cognitive neuroscience-virtual lesion, chronometry, and functional connectivity. Current opinion in neurobiology, 10(2), 232-237. https://doi.org/10.1016/s0959-4388(00)00081-7

Pasley, B. N., Allen, E. A., \& Freeman, R. D. (2009). State-dependent variability of neuronal responses to transcranial magnetic stimulation of the visual cortex. Neuron, 62(2), 291-303. https://doi.org/10.1016/j.neuron.2009.03.012

Pasternak, T., \& Greenlee, M. W. (2005). Working memory in primate sensory systems. Nature Reviews Neuroscience, 6(2), 97-107. https://doi.org/10.1038/nrn1603

Pastor, D. A., \& Lazowski, R. A. (2018). On the multilevel nature of meta-analysis: a tutorial, comparison of software programs, and discussion of analytic 
choices. Multivariate Behavioral Research, 53(1), 74-89.

https://doi.org/10.1080/00273171.2017.1365684

Peterchev, A. V., Wagner, T. A., Miranda, P. C., Nitsche, M. A., Paulus, W., Lisanby, S. H., ... \& Bikson, M. (2012). Fundamentals of transcranial electric and magnetic stimulation dose: definition, selection, and reporting practices. Brain stimulation, 5(4), 435-453. https://doi.org/10.1016/j.brs.2011.10.001

Pitcher, D., Parkin, B., \& Walsh, V. (2020). Transcranial Magnetic Stimulation and the understanding of behavior. Annual Review of Psychology, 72.

https://doi.org/10.1146/annurev-psych-081120-013144

Postle, B. R. (2006). Working memory as an emergent property of the mind and brain. Neuroscience, 139(1), 23-38. https://doi.org/10.1016/j.neuroscience.2005.06.005

Postle, B. R. (2015). The cognitive neuroscience of visual short-term memory. Current opinion in behavioral sciences, 1, 40-46. https://doi.org/10.1016/j.cobeha.2014.08.004

Postle, B. R. (2016). How does the brain keep information "in mind"?. Current Directions in Psychological Science, 25(3), 151-156. https://doi.org/10.1177\%2F0963721416643063

Prime, S. L., Vesia, M., \& Crawford, J. D. (2008). Transcranial magnetic stimulation over posterior parietal cortex disrupts transsaccadic memory of multiple objects. Journal of Neuroscience, 28(27), 6938-6949. https://doi.org/10.1523/JNEUROSCI.0542-08.2008

$\mathrm{R}$ Core Team, (2020). R: A language and environment for statistical computing. $\mathrm{R}$ Foundation for Statistical Computing, Vienna, Austria. URL https://www.R-project.org/.

Rademaker, R. L., Chunharas, C., \& Serences, J. T. (2019). Coexisting representations of sensory and mnemonic information in human visual cortex. Nature Neuroscience, 22(8), 1336-1344. https://doi.org/10.1038/s41593-019-0428-x

*Rademaker, R. L., van de Ven, V. G., Tong, F., \& Sack, A. T. (2017). The impact of early visual cortex transcranial magnetic stimulation on visual working memory precision and guess rate. PloS one, 12(4), e0175230. https://doi.org/10.1371/journal.pone.0175230

Riddle, J., Scimeca, J. M., Cellier, D., Dhanani, S., \& D’Esposito, M. (2020). Causal Evidence for a Role of Theta and Alpha Oscillations in the Control of Working Memory. Current Biology. https://doi.org/10.1016/j.cub.2020.02.065

Riley, M. R., \& Constantinidis, C. (2016). Role of prefrontal persistent activity in working memory. Frontiers in systems neuroscience, 9, 181. https://doi.org/10.3389/fnsys.2015.00181

Robertson, E. M., Theoret, H., \& Pascual-Leone, A. (2003). Studies in cognition: the problems solved and created by transcranial magnetic stimulation. Journal of cognitive neuroscience, 15(7), 948-960. https://doi.org/10.1162/089892903770007344

Robertson, E. M., Tormos, J. M., Maeda, F., \& Pascual-Leone, A. (2001). The role of the dorsolateral prefrontal cortex during sequence learning is specific for spatial information. Cerebral Cortex, 11(7), 628-635. https://doi.org/10.1093/cercor/11.7.628

Rose, N. S., LaRocque, J. J., Riggall, A. C., Gosseries, O., Starrett, M. J., Meyering, E. E., \& Postle, B. R. (2016). Reactivation of latent working memories with transcranial magnetic stimulation. Science, 354(6316), 1136-1139.

https://doi.org/10.1126/science.aah7011 
Ruff, C. C., Bestmann, S., Blankenburg, F., Bjoertomt, O., Josephs, O., Weiskopf, N., ... \& Driver, J. (2008). Distinct causal influences of parietal versus frontal areas on human visual cortex: evidence from concurrent TMS-fMRI. Cerebral cortex, 18(4), 817-827. https://doi.org/10.1093/cercor/bhm128

*Saad, E., \& Silvanto, J. (2013). How visual short-term memory maintenance modulates the encoding of external input: Evidence from concurrent visual adaptation and TMS. NeuroImage, 72, 243-251. https://doi.org/10.1016/j.neuroimage.2013.01.053

^Saad, E., Wojciechowska, M., \& Silvanto, J. (2015). Partial dissociation in the neural bases of VSTM and imagery in the early visual cortex. Neuropsychologia, 75, 143-148. https://doi.org/10.1016/j.neuropsychologia.2015.05.026

Sack, A. T. (2006). Transcranial magnetic stimulation, causal structure-function mapping and networks of functional relevance. Current opinion in neurobiology, 16(5), 593599. https://doi.org/10.1016/j.conb.2006.06.016

Sandrini, M., Umiltà, C., \& Rusconi, E. (2011). The use of transcranial magnetic stimulation in cognitive neuroscience: a new synthesis of methodological issues. Neuroscience \& Biobehavioral Reviews, 35(3), 516-536. https://doi.org/10.1016/j.neubiorev.2010.06.005

Schwarzer, G., (2007). "Meta: An R Package for Meta-Analysis.” $R$ News 7 (3): 40-45

Scimeca, J. M., Kiyonaga, A., \& D'Esposito, M. (2018). Reaffirming the sensory recruitment account of working memory. Trends in cognitive sciences, 22(3), 190-192. https://doi.org/10.1016/j.tics.2017.12.007

Siebner, H. R., Hartwigsen, G., Kassuba, T., \& Rothwell, J. C. (2009). How does transcranial magnetic stimulation modify neuronal activity in the brain? Implications for studies of cognition. Cortex, 45(9), 1035-1042. https://doi.org/10.1016/j.cortex.2009.02.007

Silva, A. E., Tsang, K., Hasan, S. J., \& Thompson, B. (2021). Precise oculocentric mapping of transcranial magnetic stimulation-evoked phosphenes. Neuroreport, 32(11), 913917. https://doi.org/10.1097/WNR.0000000000001683

Serences, J. T. (2016). Neural mechanisms of information storage in visual short-term memory. Vision research, 128, 53-67. https://doi.org/10.1016/j.visres.2016.09.010

Serences, J. T., Ester, E. F., Vogel, E. K., \& Awh, E. (2009). Stimulus-specific delay activity in human primary visual cortex. Psychological science, 20(2), 207-214. https://doi.org/10.1111/j.1467-9280.2009.02276.x

Shevlin, H. (2020). Current controversies in the cognitive science of short-term memory. Current Opinion in Behavioral Sciences, 32, 148-154. https://doi.org/10.1016/j.cobeha.2020.02.005

Silvanto, J., Bona, S., Marelli, M., \& Cattaneo, Z. (2018). On the mechanisms of Transcranial Magnetic Stimulation (TMS): How brain state and baseline performance level determine behavioral effects of TMS. Frontiers in psychology, 9, 741. https://doi.org/10.3389/fpsyg.2018.00741

$\wedge$ Silvanto, J., \& Cattaneo, Z. (2010). Transcranial magnetic stimulation reveals the content of visual short-term memory in the visual cortex. NeuroImage, 50(4), 1683-1689. https://doi.org/10.1016/j.neuroimage.2010.01.021

Silvanto, J., \& Cattaneo, Z. (2017). Common framework for "virtual lesion" and statedependent TMS: the facilitatory/suppressive range model of online TMS effects on behavior. Brain and cognition, 119, 32-38. https://doi.org/10.1016/j.bandc.2017.09.007 
${ }^{\wedge}$ Silvanto, J., \& Soto, D. (2012). Causal evidence for subliminal percept-to-memory interference in early visual cortex. NeuroImage, 59(1), 840-845. https://doi.org/10.1016/j.neuroimage.2011.07.062

Song, J. H., \& Jiang, Y. (2006). Visual working memory for simple and complex features: An fMRI study. Neuroimage, 30(3), 963-972. https://doi.org/10.1016/j.neuroimage.2005.10.006

^Soto, D., Llewelyn, D., \& Silvanto, J. (2012). Distinct causal mechanisms of attentional guidance by working memory and repetition priming in early visual cortex. The Journal of Neuroscience : The Official Journal of the Society for Neuroscience, 32(10), 34473452. https://doi.org/10.1523/jneurosci.6243-11.2012

Sprague, T. C., Saproo, S., \& Serences, J. T. (2015). Visual attention mitigates information loss in small-and large-scale neural codes. Trends in Cognitive Sciences, 19(4), 215-226. https://doi.org/10.1016/j.tics.2015.02.005

Sreenivasan, K. K., Curtis, C. E., \& D'Esposito, M. (2014). Revising the role of persistent neural activity during working memory. Trends in Cognitive Sciences, 18(2), 82-89. https://doi.org/10.1016/j.tics.2013.12.001

Sreenivasan, K. K., \& D'Esposito, M. (2019). The what, where and how of delay activity. Nature Reviews Neuroscience, 20(8), 466-481. https://doi.org/10.1038/s41583019-0176-7

Stokes, M. G. (2015). 'Activity-silent'working memory in prefrontal cortex: a dynamic coding framework. Trends in cognitive sciences, 19(7), 394-405. https://doi.org/10.1016/j.tics.2015.05.004

Supèr, H., Spekreijse, H., \& Lamme, V. A. (2001). Two distinct modes of sensory processing observed in monkey primary visual cortex (V1). Nature neuroscience, 4(3), 304-310. https://doi.org/10.1038/85170

Tapia, E., \& Beck, D. M. (2014). Probing feedforward and feedback contributions to awareness with visual masking and transcranial magnetic stimulation. Frontiers in Psychology, 5. https://doi.org/10.3389/fpsyg.2014.01173

Tcheslavski, G. V., Vasefi, M., \& Gonen, F. F. (2018). Response of a human visual system to continuous color variation: An EEG-based approach. Biomedical Signal Processing and Control, 43, 130-137. https://doi.org/10.1016/j.bspc.2018.03.001

ten Oever, S., De Weerd, P., \& Sack, A. T. (2020). Phase-dependent amplification of working memory content and performance. Nature communications, 11(1), 1-8. https://doi.org/10.1038/s41467-020-15629-7

Teng, C., \& Postle, B. R. (2021). Understanding occipital and parietal contributions to visual working memory: Commentary on Xu (2020). Visual Cognition, 1-8. https://doi.org/10.1080/13506285.2021.1883171

Todd, J. J., \& Marois, R. (2004). Capacity limit of visual short-term memory in human posterior parietal cortex. Nature, 428, 751-754. https://doi.org/10.1038/nature02466

Tong, F., Meng, M., \& Blake, R. (2006). Neural bases of binocular rivalry. Trends in cognitive sciences, 10(11), 502-511. https://doi.org/10.1016/j.tics.2006.09.003

van de Ven, V., \& Sack, A. T. (2013). Transcranial magnetic stimulation of visual cortex in memory: Cortical state, interference and reactivation of visual content in memory. Behavioural Brain Research, 236(1), 67-77. https://doi.org/10.1016/j.bbr.2012.08.001

*van de Ven, V., Jacobs, C., \& Sack, A. T. (2012). Topographic contribution of early visual cortex to short-term memory consolidation: A transcranial magnetic stimulation study. 
Journal of Neuroscience, 32(1), 4-11. https://doi.org/10.1523/JNEUROSCI.3261$\underline{11.2012}$

Van Kerkoerle, T., Self, M. W., \& Roelfsema, P. R. (2017). Layer-specificity in the effects of attention and working memory on activity in primary visual cortex. Nature communications, 8(1), 1-14. https://doi.org/10.1038/ncomms13804

*van Lamsweerde, A. E., \& Johnson, J. S. (2017). Assessing the effect of early visual cortex Transcranial Magnetic Stimulation on working memory consolidation. Journal of Cognitive Neuroscience, 29(7), 1226. https://doi.org/10.1162/jocn_a 01113

Vogel, E. K., \& Machizawa, M. G. (2004). Neural activity predicts individual differences in visual working memory capacity. Nature, 428(6984), 748-751. https://doi.org/10.1038/nature02447

Vogel, E. K., McCollough, A. W., \& Machizawa, M. (2005). Neural measures reveal individual differences in controlling access to working memory. Nature, 438, 500-503. https://doi.org/10.1038/nature04171

Vogel, E. K., Woodman, G. F., \& Luck, S. J. (2001). Storage of features, conjunctions, and objects in visual working memory. Journal of experimental psychology: human perception and performance, 27(1), 92. https://doi.org/10.1037/0096-1523.27.1.92

Vogel, E. K., Woodman, G. F., \& Luck, S. J. (2006). The time course of consolidation in visual working memory. Journal of Experimental Psychology: Human Perception and Performance, 32(6), 1436. https://doi.org/10.1037/0096-1523.32.6.1436

Walsh, V., \& Pascual-Leone, A. (2003). Transcranial magnetic stimulation: a neurochronometrics of mind. MIT press. https://doi.org/10.7551/mitpress/6896.001.0001

Wichmann, W., \& Müller-Forell, W. (2004). Anatomy of the visual system. European journal of radiology, 49(1), 8-30. https://doi.org/10.1016/j.ejrad.2003.11.001

$\mathrm{Xu}, \mathrm{Y}$. (2007). The role of the superior intraparietal sulcus in supporting visual short-term memory for multifeature objects. Journal of Neuroscience, 27(43), 11676-11686. https://doi.org/10.1523/JNEUROSCI.3545-07.2007

$\mathrm{Xu}, \mathrm{Y}$. (2017). Reevaluating the sensory account of visual working memory storage. Trends in Cognitive Sciences, 21(10), 794-815. https://doi.org/10.1016/j.tics.2017.06.013

$\mathrm{Xu}, \mathrm{Y}$. (2018). Sensory cortex is nonessential in working memory storage. Trends in cognitive sciences, 22(3), 192-193. https://doi.org/10.1016/j.tics.2017.12.008

$\mathrm{Xu}, \mathrm{Y}$. (2020). Revisit once more the sensory storage account of visual working memory. Visual Cognition, 1-14. https://doi.org/10.1080/13506285.2020.1818659

$\mathrm{Xu}, \mathrm{Y}$. (2021). Towards a better understanding of information storage in visual working memory. Visual Cognition, 1-9. https://doi.org/10.1080/13506285.2021.1946230

Xu, Y., \& Chun, M. M. (2006). Dissociable neural mechanisms supporting visual short-term memory for objects. Nature, 440(7080), 91-95. https://doi.org/10.1038/nature04262

Ye, C., Liang, T., Zhang, Y., Xu, Q., Zhu, Y., \& Liu, Q. (2020). The two-stage process in visual working memory consolidation. Scientific Reports, 10(1), 1-11. https://doi.org/10.1038/s41598-020-70418-y

Ye, C., Hu, Z., Li, H., Ristaniemi, T., Liu, Q., \& Liu, T. (2017). A two-phase model of resource allocation in visual working memory. Journal of Experimental Psychology: Learning, Memory, and Cognition, 43(10), 1557. https://doi.apa.org/doi/10.1037/xlm0000376 
Zhao, Y. J., Kay, K. N., Tian, Y., \& Ku, Y. (2021). Sensory Recruitment Revisited: Ipsilateral V1 Involved in Visual Working Memory. Cerebral Cortex. https://doi.org/10.1093/cercor/bhab300

Zhang, W., \& Luck, S. J. (2008). Discrete fixed-resolution representations in visual working memory. Nature, 453(7192), 233-235. https://doi.org/10.1038/nature06860

Zhang, J., Ye, C., Sun, H. J., Zhou, J., Liang, T., Li, Y., \& Liu, Q. (2021). The passive state: A protective mechanism for information in working memory tasks. Journal of Experimental Psychology: Learning, Memory, and Cognition. https://doi.apa.org/doi/10.1037/xlm0001092

*Zokaei, N., Manohar, S., Husain, M., \& Feredoes, E. (2014). Causal evidence for a privileged working memory state in early visual cortex. Journal of Neuroscience, 34(1), 158-162. https://doi.org/10.1523/jneurosci.2899-13.2014 


\section{Figures and Tables}

Figure 1: The study's PRISMA statement.

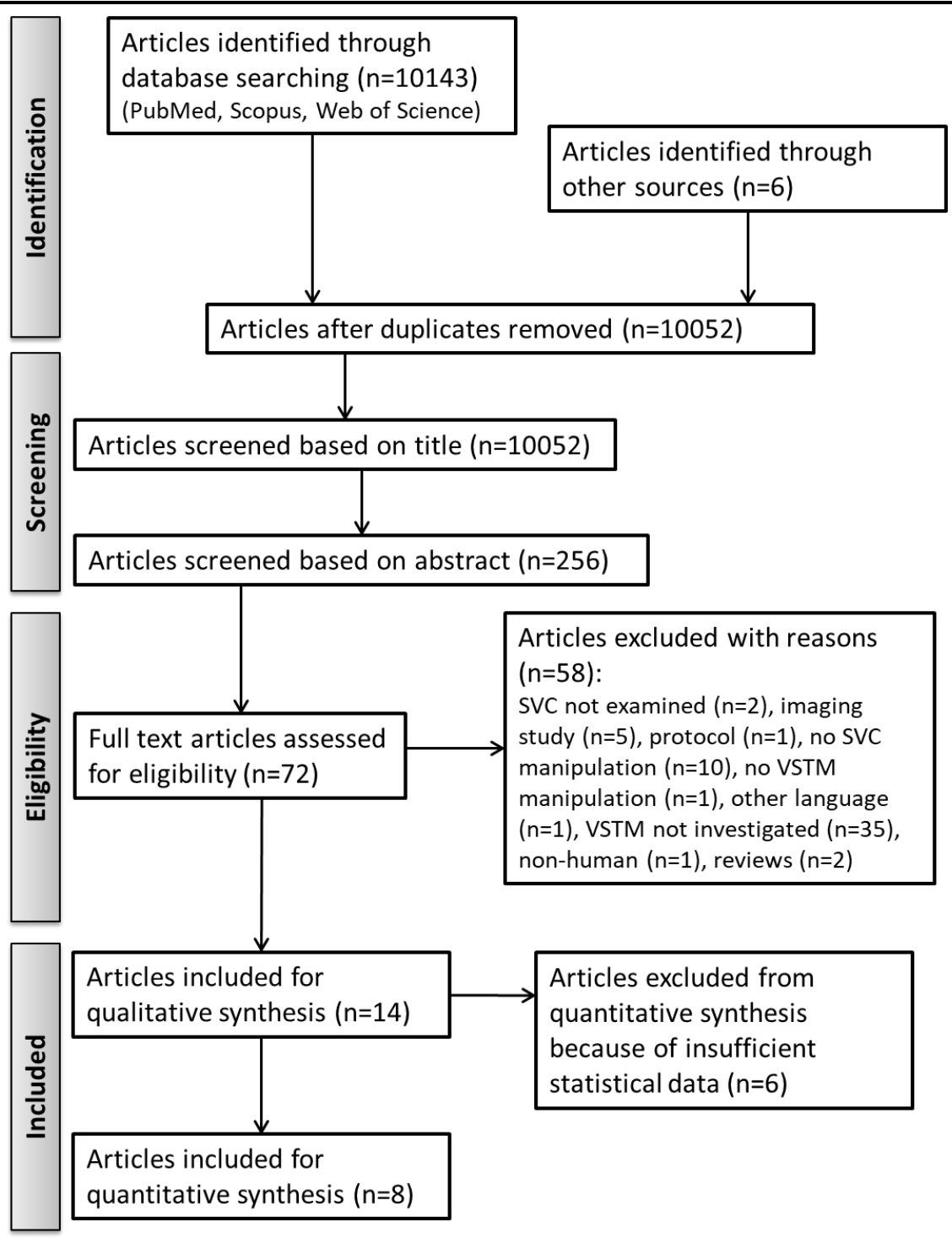

Fig 1. The PRISMA flow diagram followed for the systematic review and meta-analyses as suggested by Moher et al. (2009). Screening and eligibility assessment were completed by two independent researchers. SVC; sensory visual cortex, VSTM; visual short-term memory. 
Table 1: Articles systematically identified to be included in the systematic review and meta-analysis.

\begin{tabular}{|c|c|c|c|c|c|c|c|c|c|}
\hline & ARTICLE & $\begin{array}{l}\text { DESCRIPTI } \\
\text { ON }\end{array}$ & $\begin{array}{c}\text { ROI } \\
\text { (PULSE) }\end{array}$ & $\begin{array}{c}\text { TARGETING (OUTPUT } \\
\text { POWER) }\end{array}$ & TASK & CONTROL & $\begin{array}{l}\text { TMS TEMPORAL } \\
\text { POINT } \\
\text { (AFTER STIMULUS } \\
\text { OFFSET) }\end{array}$ & $\mathbf{N}$ & $\begin{array}{l}\text { BEHAVIORAL } \\
\text { MEASURE }\end{array}$ \\
\hline 1 & $\begin{array}{l}\text { Cattaneo et } \\
\text { al. (2009) }\end{array}$ & $\begin{array}{l}\text { SVC role in } \\
\text { mental- } \\
\text { imagery and } \\
\text { short-term } \\
\text { memory }\end{array}$ & V1 (sp) & $\begin{array}{l}\text { Phosphene induction } \\
\text { ( } 65 \% \text { of Magstim } 200 \\
\text { stimulator) }\end{array}$ & $\begin{array}{l}\text { Time Memory or } \\
\text { imagery task }\end{array}$ & $\begin{array}{l}\text { No TMS \& } \\
\text { vertex TMS }\end{array}$ & $\begin{array}{l}\text { Exp1: } 2000 \mathrm{~ms} \\
\text { Exp2: } 0 \mathrm{~ms}\end{array}$ & $\begin{array}{l}\text { Exp1: } \\
14 \\
\text { Exp2: } \\
14\end{array}$ & Accuracy \\
\hline 2 & $\begin{array}{l}\text { Silvanto et al. } \\
(2010)\end{array}$ & $\begin{array}{l}\text { SVC role in } \\
\text { VSTM }\end{array}$ & $\begin{array}{l}\text { Right or } \\
\text { left } \\
\text { V5/MT+ } \\
(\mathrm{sp})\end{array}$ & $\begin{array}{l}\text { Phosphene induction } \\
\text { (120\% of phosphene } \\
\text { threshold) }\end{array}$ & $\begin{array}{l}\text { Exp1: motion } \\
\text { speed detection } \\
\text { task }\end{array}$ & $\begin{array}{l}\text { Ipsi- } \\
\text { /contra- \& } \\
\text { No TMS }\end{array}$ & $3000 \mathrm{~ms}$ & 9 & Accuracy \\
\hline 3 & $\begin{array}{l}\text { Cattaneo et } \\
\text { al. (2012) }\end{array}$ & $\begin{array}{l}\text { SVC role in } \\
\text { mental } \\
\text { imagery }\end{array}$ & V1 (3p) & $\begin{array}{l}2 \mathrm{~cm} \text { above inion }(60 \% \text { of } \\
\text { Magstim SuperRapid } \\
\text { stimulator) }\end{array}$ & $\begin{array}{l}\text { Exp2: Time } \\
\text { imagery task }\end{array}$ & $\begin{array}{l}\text { No TMS \& } \\
\text { vertex TMS }\end{array}$ & $1000 \mathrm{~ms}$ & 10 & Accuracy \\
\hline 4 & $\begin{array}{l}\text { Soto et al. } \\
(2012)\end{array}$ & $\begin{array}{l}\text { SVC role in } \\
\text { attentional } \\
\text { guidance by } \\
\text { priming and } \\
\text { working } \\
\text { memory }\end{array}$ & V1 (3p) & $\begin{array}{l}2 \mathrm{~cm} \text { above inion }(90 \% \text { of } \\
\text { phosphene threshold) }\end{array}$ & $\begin{array}{l}\text { Priming } \\
\text { detection task, } \\
\text { with working } \\
\text { memory task }\end{array}$ & Sham TMS & $1700 \mathrm{~ms}$ & 12 & Accuracy \\
\hline 5 & $\begin{array}{l}\text { Silvanto et al. } \\
(2012)\end{array}$ & $\begin{array}{l}\text { Subliminal } \\
\text { perception } \\
\text { interference } \\
\text { in memory }\end{array}$ & V1 (3p) & $\begin{array}{l}\text { Phosphene induction } \\
(90 \% \text { of phosphene } \\
\text { threshold for } n=7 \text {, } \\
50 \% \text { of Medtronic } \\
\text { MagPro R } 30 \text { stimulator } \\
\text { for } n=3)\end{array}$ & $\begin{array}{l}\text { Exp1b: } \\
\text { Orientation } \\
\text { change detection } \\
\text { task }\end{array}$ & Sham TMS & $1000 \mathrm{~ms}$ & $\begin{array}{l}\text { Exp1b: } \\
10\end{array}$ & $\begin{array}{l}\text { Detection } \\
\text { sensitivity }\end{array}$ \\
\hline 6 & $\begin{array}{l}\text { van de Ven et } \\
\text { al. }(2012)\end{array}$ & $\begin{array}{l}\text { SVC role in } \\
\text { VSTM }\end{array}$ & $\begin{array}{l}\text { Right or } \\
\text { left V1 (sp) }\end{array}$ & $\begin{array}{l}\text { (1) Phosphene induction } \\
\text { for } n=8 \\
\text { (2) Neuronavigation for } \\
n=5 \text { ( } 110 \% \text { of phosphene } \\
\text { threshold) }\end{array}$ & $\begin{array}{l}\text { Exp2: Non- } \\
\text { natural shape } \\
\text { change-detection } \\
\text { task }\end{array}$ & $\begin{array}{l}\text { Ipsi- } \\
\text { /contra- }\end{array}$ & $\begin{array}{l}100 \mathrm{~ms}, 200 \mathrm{~ms} \text { or } \\
400 \mathrm{~ms}\end{array}$ & 12 & $\begin{array}{l}\text { Detection } \\
\text { sensitivity }\end{array}$ \\
\hline
\end{tabular}




\section{Table 1 Continued}

\begin{tabular}{|c|c|c|c|c|c|c|c|c|}
\hline $\begin{array}{l}\text { Saad et al. } \\
(2013)\end{array}$ & $\begin{array}{l}\text { Effects of } \\
\text { external } \\
\text { visual input } \\
\text { in internal } \\
\text { representatio } \\
\text { ns }\end{array}$ & V1 (5p) & $\begin{array}{l}2 \mathrm{~cm} \text { above and } .5 \mathrm{~cm} \\
\text { laterally from the inion } \\
\text { towards the right } \\
\text { hemisphere } \\
\text { ( } 45 \% \text { of a Nexstim } \\
\text { stimulator) }\end{array}$ & $\begin{array}{l}\text { Exp2: } \\
\text { Orientation } \\
\text { change detection } \\
\text { task } \\
\text { Exp3: Shape } \\
\text { change detection } \\
\text { task }\end{array}$ & Sham TMS & $\begin{array}{l}\text { Exp2: } 2000 \mathrm{~ms} \text { or } \\
\text { 5000 ms } \\
\text { Exp3: } 2000 \mathrm{~ms}\end{array}$ & $\begin{array}{l}\text { Exp2: } \\
16 \\
\text { Exp3: } 8\end{array}$ & Accuracy \\
\hline $\begin{array}{l}\text { Zokaei et al. } \\
(2014)\end{array}$ & $\begin{array}{l}\text { Effects of } \\
\text { TMS } \\
\text { depending on } \\
\text { representatio } \\
\text { n state in } \\
\text { VSTM }\end{array}$ & $\begin{array}{l}\text { V5/MT+ } \\
(4 \mathrm{p})\end{array}$ & $\begin{array}{l}\text { fMRI localization ( } 60 \% \\
\text { of Magstim Rapid }{ }^{2} \\
\text { stimulator) }\end{array}$ & $\begin{array}{l}\text { Motion match to } \\
\text { sample task }\end{array}$ & $\begin{array}{l}\text { Exp1: low } \\
\text { power TMS } \\
\text { Epx2: } \\
\text { Low power } \\
\text { TMS \& } \\
\text { Vertex } \\
\text { TMS }\end{array}$ & $\begin{array}{l}\text { Exp1: } 3100 \mathrm{~ms} \\
\text { Exp2: } 300 \mathrm{~ms} \\
\text { after first or } \\
\text { second memory } \\
\text { array }\end{array}$ & $\begin{array}{l}\text { Exp1: } \\
13 \\
\text { Exp2: } \\
17\end{array}$ & Precision \\
\hline $\begin{array}{l}\text { Malik et al. } \\
(2015)\end{array}$ & $\begin{array}{l}\text { SVC role in } \\
\text { trans- } \\
\text { saccadic } \\
\text { memory of } \\
\text { features }\end{array}$ & $\begin{array}{l}\text { Right and } \\
\text { left V1 (3p) }\end{array}$ & $\begin{array}{l}\text { Neuronavigation }(60 \% \text { of } \\
\text { N/A stimulator) }\end{array}$ & $\begin{array}{l}\text { Fixation task: } \\
\text { VSTM } \\
\text { orientation } \\
\text { change detection } \\
\text { task }\end{array}$ & $\begin{array}{l}\text { Ipsi- } \\
\text { /contra- \& } \\
\text { no TMS }\end{array}$ & $250 \mathrm{~ms}$ & 8 & Percent correct \\
\hline $\begin{array}{l}\text { Saad et al. } \\
(2015)\end{array}$ & $\begin{array}{l}\text { Difference in } \\
\text { VSTM and } \\
\text { imagery } \\
\text { neural bases }\end{array}$ & V1 (5p) & $\begin{array}{l}\text { (1) Neuronavigation for } \\
\mathrm{n}=\mathrm{N} / \mathrm{A} \\
\text { (2) Phosphene induction } \\
\text { for } \mathrm{n}=\mathrm{N} / \mathrm{A} \\
(90 \% \text { of phosphene } \\
\text { threshold for } \mathrm{n}=\mathrm{N} / \mathrm{A} \text { or } \\
65 \% \text { of Magstim Rapid } \\
\text { stimulator for } \mathrm{n}=\mathrm{N} / \mathrm{A})\end{array}$ & $\begin{array}{l}\text { Orientation } \\
\text { change detection } \\
\text { task }\end{array}$ & Sham TMS & $2600 \mathrm{~ms}$ & 15 & $\begin{array}{l}\text { Detection } \\
\text { sensitivity }\end{array}$ \\
\hline $\begin{array}{l}\text { Koivisto et } \\
\text { al. (2017) }\end{array}$ & $\begin{array}{l}\text { TMS effects } \\
\text { on quality of } \\
\text { memory } \\
\text { representatio } \\
\text { ns }\end{array}$ & $\begin{array}{l}\text { Right and } \\
\text { left V1 (sp) }\end{array}$ & $\begin{array}{l}\text { Neuronavigation }(65 \% \text { of } \\
\text { Nextim eXimia }^{\mathrm{m}} \\
\text { stimulator) }\end{array}$ & $\begin{array}{l}\text { VSTM } \\
\text { orientation } \\
\text { match task }\end{array}$ & $\begin{array}{l}\text { Exp1: Ipsi- } \\
\text { / contra- \& } \\
\text { no TMS } \\
\text { Exp2: } \\
\text { Ipsi-/ } \\
\text { contra- \& } \\
\text { no TMS }\end{array}$ & $\begin{array}{l}\text { Exp1: }-30 \mathrm{~ms} \text { to } \\
120 \mathrm{~ms}(30 \mathrm{~ms} \\
\text { intervals) } \\
\text { Exp2: } 120 \mathrm{~ms} \text {, or } \\
150 \mathrm{~ms} \text {, or } 180 \mathrm{~ms}\end{array}$ & $\begin{array}{l}\text { Exp1: } \\
12 \\
\text { Exp2: } 7\end{array}$ & $\begin{array}{l}\text { (1) Proportion of } \\
\text { guessing } \\
\text { (2) Precision }\end{array}$ \\
\hline
\end{tabular}




\section{Table 1 Continued} al. (2017) VSTM

van

13 Lamsweerde et al. (2017) VSTM
SVC role in

\section{Training role}

14 Jia et al. (2021)
in VSTM
Right or
representatio

Right or

Neuronavigation $(80 \%$ of

left V1 (3p) phosphene threshold)

VSTM

orientation match task

Ipsi-

/contra- \&

Sham TMS

(1) Absolute error

(2) Precision

(3) Guess

frequency

(1) Absolute error

Exp3: colour match to sample

Exp3: ipsi-/ Exp3: $0 \mathrm{~ms}, 100$ of phosphene threshold) contra-
(2) Precision

(3) Guess

frequency

(4) Swap errors

Tab 1. The included papers with short descriptions that were identified after following the PRISMA statement for a systematic approach in literature search

Abbreviations: 3p; three pulses, 5p; five pulses, Exp; experiment, ROI; region of interest, sp; single pulse, TMS; transcranial magnetic stimulation, VSTM; visual short-term memory 
Table 2: Experiments included in the meta-analysis of TMS on SVC during the encoding phase of a VSTM task.

\begin{tabular}{|c|c|c|c|c|c|c|c|c|}
\hline & STUDY & $\begin{array}{l}\text { EFFECT } \\
\text { SIZE }\end{array}$ & $\begin{array}{l}\text { STANDARD } \\
\text { ERROR }\end{array}$ & $\begin{array}{c}\text { Z- } \\
\text { VALUE }\end{array}$ & $\begin{array}{c}\text { P- } \\
\text { VALUE }\end{array}$ & $\mathbf{N}$ & $\begin{array}{l}\text { BEHAVIORAL } \\
\text { MEASURE } \\
\text { (condition) }\end{array}$ & $\begin{array}{l}\text { CONTROL } \\
\text { CONDITION }\end{array}$ \\
\hline 1 & $\begin{array}{l}\text { Cattaneo et al } \\
(2009) \mathrm{a}\end{array}$ & 0.82 & 0.32 & 2.55 & 0.01 & 14 & Accuracy & No TMS \\
\hline 2 & $\begin{array}{l}\text { Cattaneo et al } \\
(2009) \mathrm{b}\end{array}$ & 0.64 & 0.30 & 2.11 & 0.03 & 14 & Accuracy & Sham TMS \\
\hline 3 & $\begin{array}{l}\text { van de Ven et } \\
\text { al (2012)a }\end{array}$ & 0.36 & 0.31 & 1.16 & 0.25 & 12 & $\begin{array}{c}\text { Detection Sensitivity } \\
A^{\prime}(\mathrm{LL})\end{array}$ & Ipsilateral SVC \\
\hline 4 & $\begin{array}{l}\text { van de Ven et } \\
\text { al (2012)b }\end{array}$ & 0.23 & 0.31 & 0.75 & 0.46 & 12 & $\begin{array}{c}\text { Detection Sensitivity } \\
A^{\prime}(\mathrm{HL})\end{array}$ & Ipsilateral SVC \\
\hline 5 & $\begin{array}{l}\text { van de Ven et } \\
\text { al (2012)c }\end{array}$ & 0.00 & 0.31 & 0.00 & 1.00 & 12 & $\begin{array}{c}\text { Detection Sensitivity } \\
A^{\prime}(\mathrm{LL})\end{array}$ & Ipsilateral SVC \\
\hline 6 & $\begin{array}{l}\text { van de Ven et } \\
\text { al (2012)d }\end{array}$ & 0.97 & 0.34 & 2.82 & $<0.01$ & 12 & $\begin{array}{c}\text { Detection Sensitivity } \\
A^{\prime}(\mathrm{HL})\end{array}$ & Ipsilateral SVC \\
\hline 7 & $\begin{array}{l}\text { Koivisto et al } \\
(2017) \mathrm{a}\end{array}$ & 3.45 & 0.65 & 5.35 & $<0.01$ & 12 & $\begin{array}{l}\text { Proportion of Guess } \\
\text { (Exp1 } 60 \mathrm{~ms})\end{array}$ & No TMS \\
\hline 8 & $\begin{array}{l}\text { Koivisto et al } \\
(2017) \mathrm{b}\end{array}$ & 3.81 & 0.69 & 5.49 & $<0.01$ & 12 & $\begin{array}{l}\text { Proportion of Guess } \\
\quad \text { (Exp1 } 90 \mathrm{~ms} \text { ) }\end{array}$ & No TMS \\
\hline 9 & $\begin{array}{l}\text { Koivisto et al } \\
(2017) \mathrm{c}\end{array}$ & 3.15 & 0.60 & 5.28 & $<0.01$ & 12 & $\begin{array}{l}\text { Proportion of Guess } \\
\quad \text { (Exp1 } 120 \mathrm{~ms})\end{array}$ & No TMS \\
\hline 10 & $\begin{array}{l}\text { Koivisto et al } \\
(2017) \mathrm{d}\end{array}$ & 0.42 & 0.43 & 0.97 & 0.33 & 7 & $\begin{array}{l}\text { Proportion of Guess } \\
\quad \text { (Exp2 } 120 \mathrm{~ms})\end{array}$ & Ipsilateral SVC \\
\hline 11 & $\begin{array}{l}\text { Koivisto et al } \\
\text { (2017)e }\end{array}$ & 0.64 & 0.45 & 1.43 & 0.15 & 7 & $\begin{array}{l}\text { Proportion of Guess } \\
\quad \text { (Exp2 } 150 \mathrm{~ms})\end{array}$ & Ipsilateral SVC \\
\hline 12 & $\begin{array}{l}\text { Koivisto et al } \\
(2017) \mathrm{f}\end{array}$ & 0.09 & 0.42 & 0.21 & 0.83 & 7 & $\begin{array}{l}\text { Proportion of Guess } \\
\quad \text { (Exp3 } 150 \mathrm{~ms})\end{array}$ & Ipsilateral SVC \\
\hline 13 & $\begin{array}{l}\text { Koivisto et al } \\
(2017) \mathrm{g}\end{array}$ & 0.22 & 0.42 & 0.52 & 0.60 & 7 & $\begin{array}{l}\text { Proportion of Guess } \\
\text { (Exp2 } 120 \mathrm{~ms})\end{array}$ & No TMS \\
\hline 14 & $\begin{array}{l}\text { Koivisto et al } \\
(2017) \mathrm{h}\end{array}$ & 0.50 & 0.44 & 1.15 & 0.25 & 7 & $\begin{array}{l}\text { Proportion of Guess } \\
\quad(\text { Exp2 } 150 \mathrm{~ms})\end{array}$ & No TMS \\
\hline 15 & $\begin{array}{l}\text { Koivisto et al } \\
\text { (2017)i }\end{array}$ & 0.06 & 0.42 & 0.14 & 0.89 & 7 & $\begin{array}{l}\text { Proportion of Guess } \\
\quad \text { (Exp3 } 150 \mathrm{~ms} \text { ) }\end{array}$ & No TMS \\
\hline 16 & $\begin{array}{l}\text { Rademaker et } \\
\text { al (2017) }\end{array}$ & 0.32 & 0.39 & 0.81 & 0.42 & 8 & Absolute Error & Sham TMS \\
\hline 17 & $\begin{array}{l}\text { van } \\
\text { Lamsweerde et } \\
\text { al (2017)a }\end{array}$ & 0.11 & 0.22 & 0.49 & 0.62 & 21 & Guess Rate $(100 \mathrm{~ms})$ & Ipsilateral SVC \\
\hline 18 & $\begin{array}{l}\text { van } \\
\text { Lamsweerde et } \\
\text { al (2017)b }\end{array}$ & 0.56 & 0.24 & 2.34 & 0.02 & 21 & Guess Rate $(0 \mathrm{~ms})$ & Ipsilateral SVC \\
\hline
\end{tabular}


Figure 2: Meta-analyses of SVC TMS on VSTM performance.

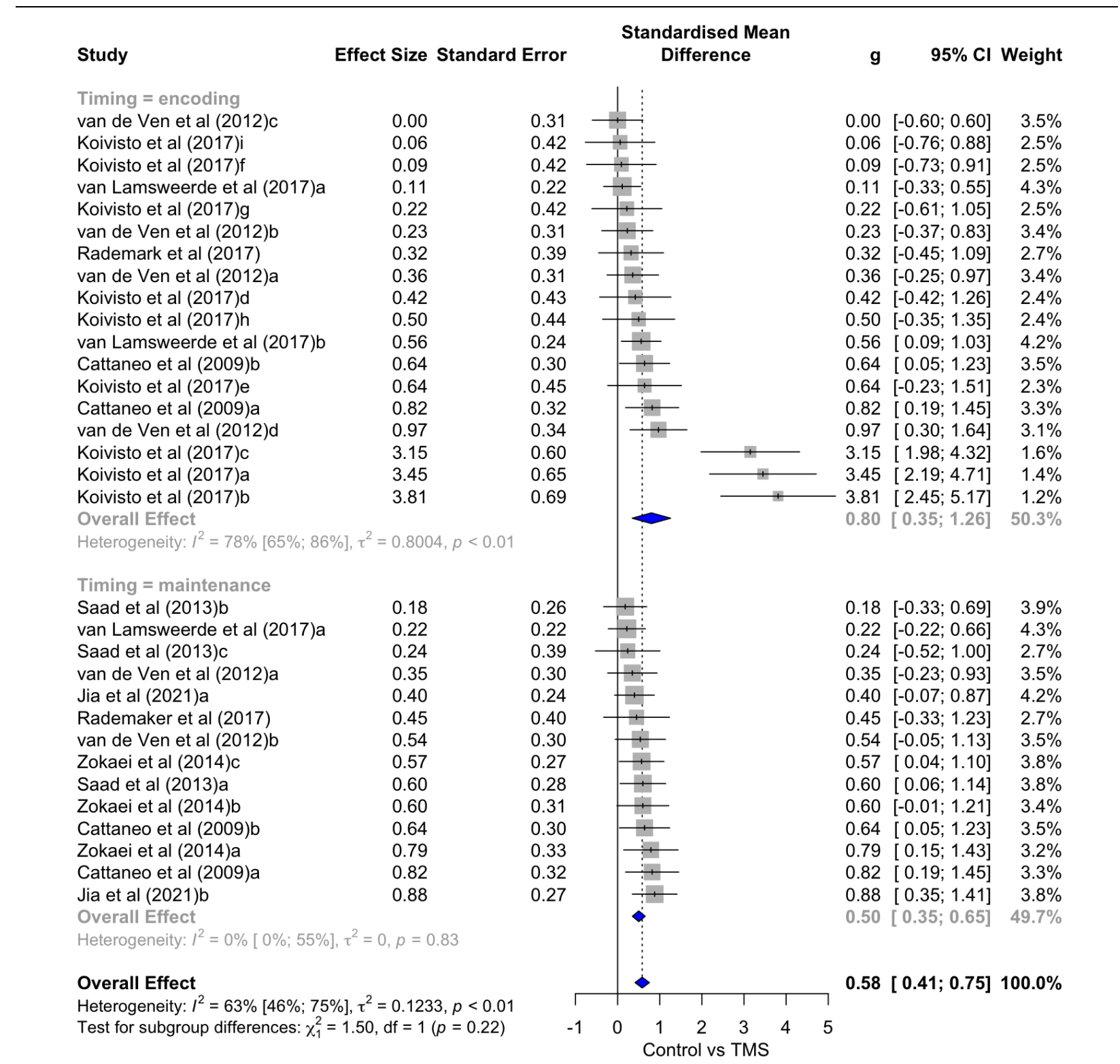

Fig 2. The meta-analyses of behavioral outcomes when inducing TMS on the SVC during a VSTM task. The forest plot of a random effects model pooling the effect sizes of experiments inducing TMS on SVC during the encoding phase (top) and maintenance phase (bottom) of a VSTM task. In both meta-analyses the overall standard means difference indicates that TMS does have an effect on behavior when induced on the SVC, and these two overall effects do not differ between them. An overall effect including all studies (both encoding and maintenance) show a significantly moderate effect of TMS. SVC; sensory visual cortex, TMS; transcranial magnetic stimulation, VSTM; visual short-term memory. 
Figure 3: Funnel plots of the identified studies.

A

Encoding:

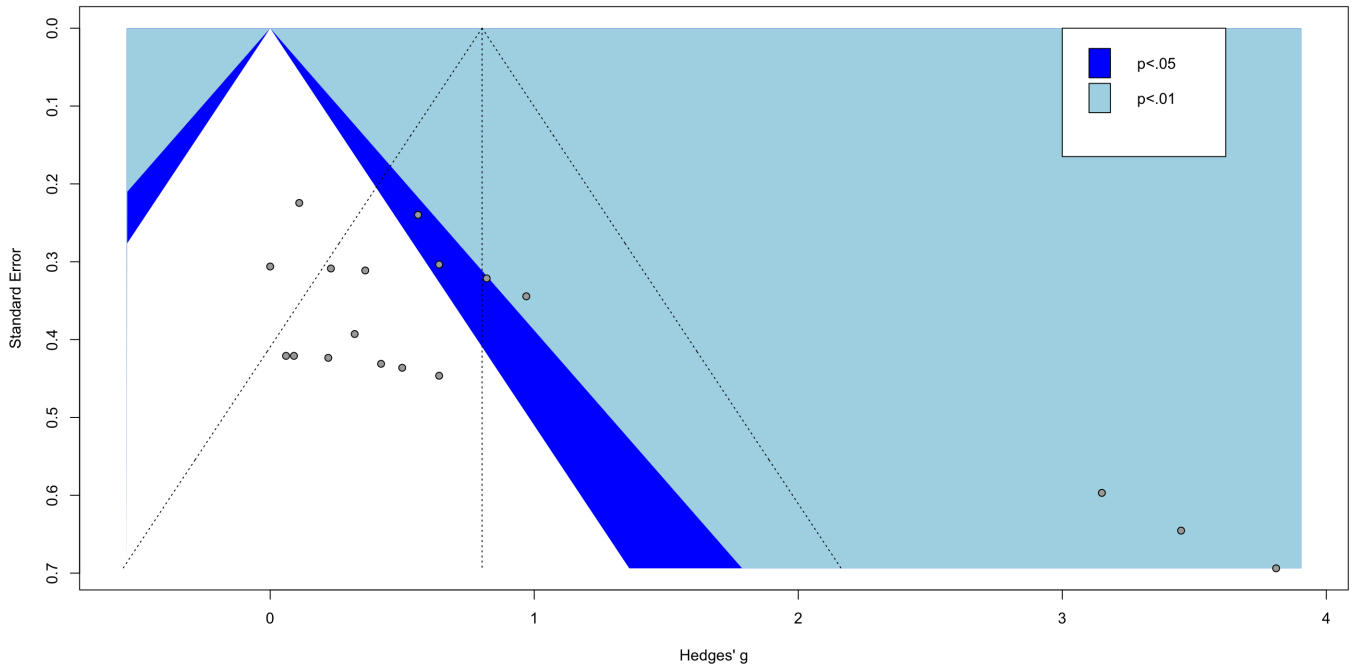

Maintenance:

B

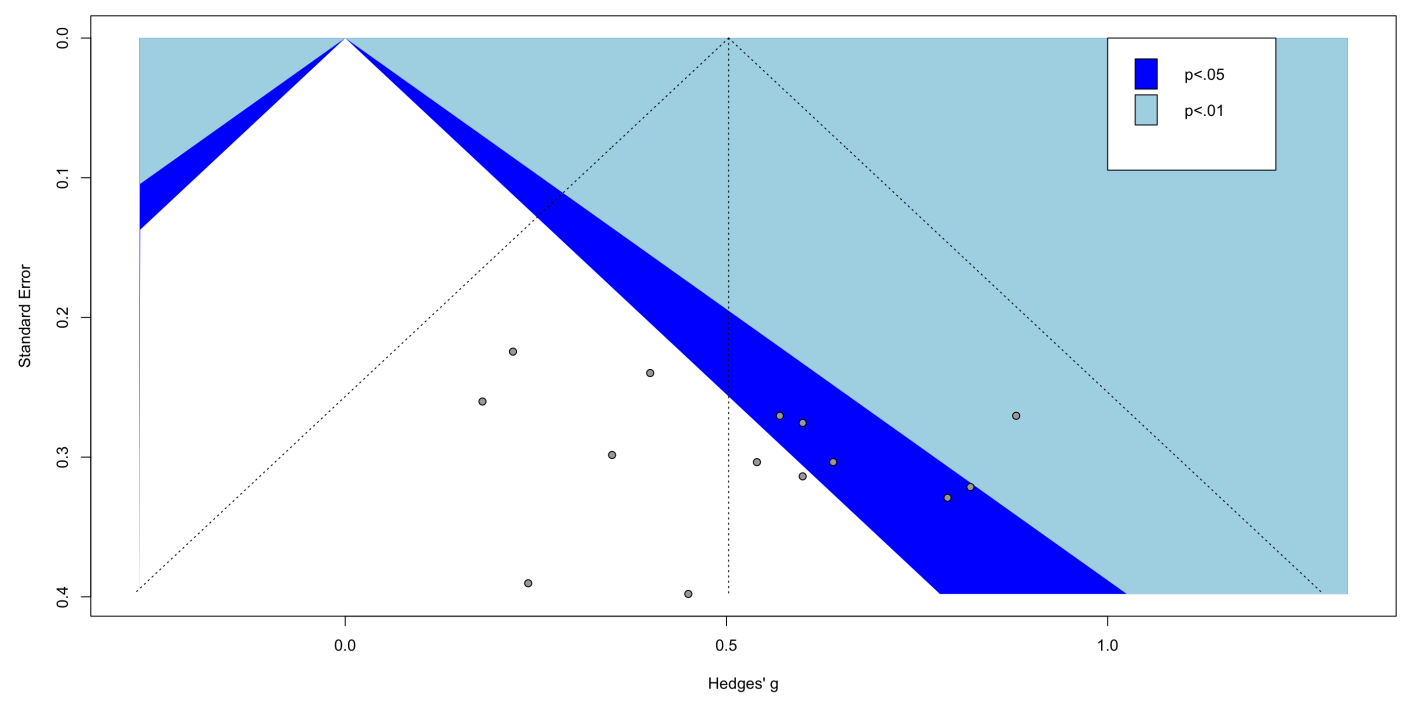

Fig 3. The funnel plots of the identified experiments in (A) encoding and (B) maintenance to investigate small study bias. An asymmetry is evident in the (A) encoding meta-analysis indicating publication bias, however, (B) the maintenance funnel plot appears symmetrical. 
Figure 4: The three-level meta-analyses models.

Total Variance: 0.358

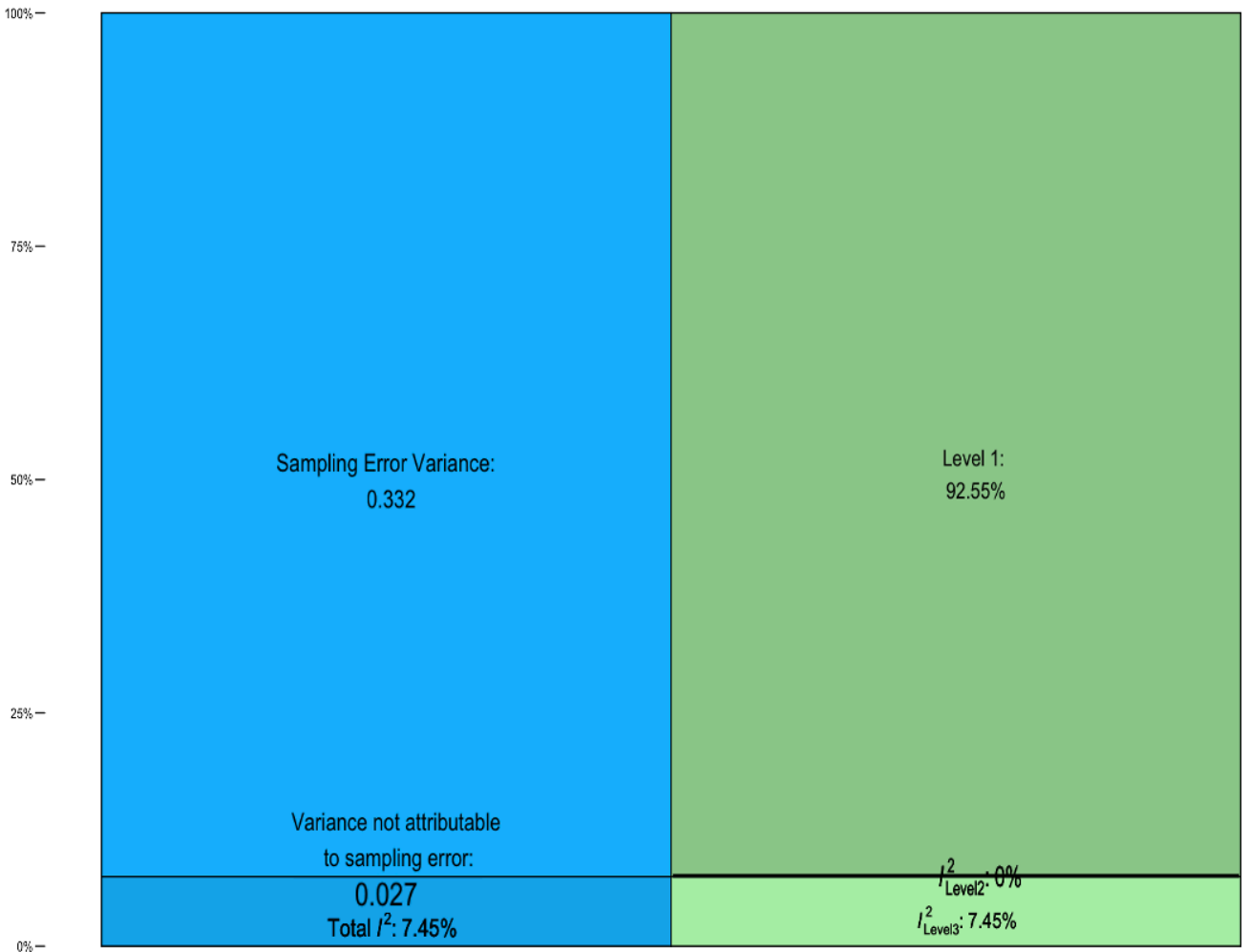

Fig 4. The distribution of variance in the three-model meta-analysis, where experiments were clustered to their corresponding studies to introduce the study level (level 2) in the meta-analyses. No variance was explained by level 2 in the three-level models. VSTM; visual short-term memory. 
Figure 5: Meta-regression of SVC TMS on VSTM performance predicted by TMS timing.

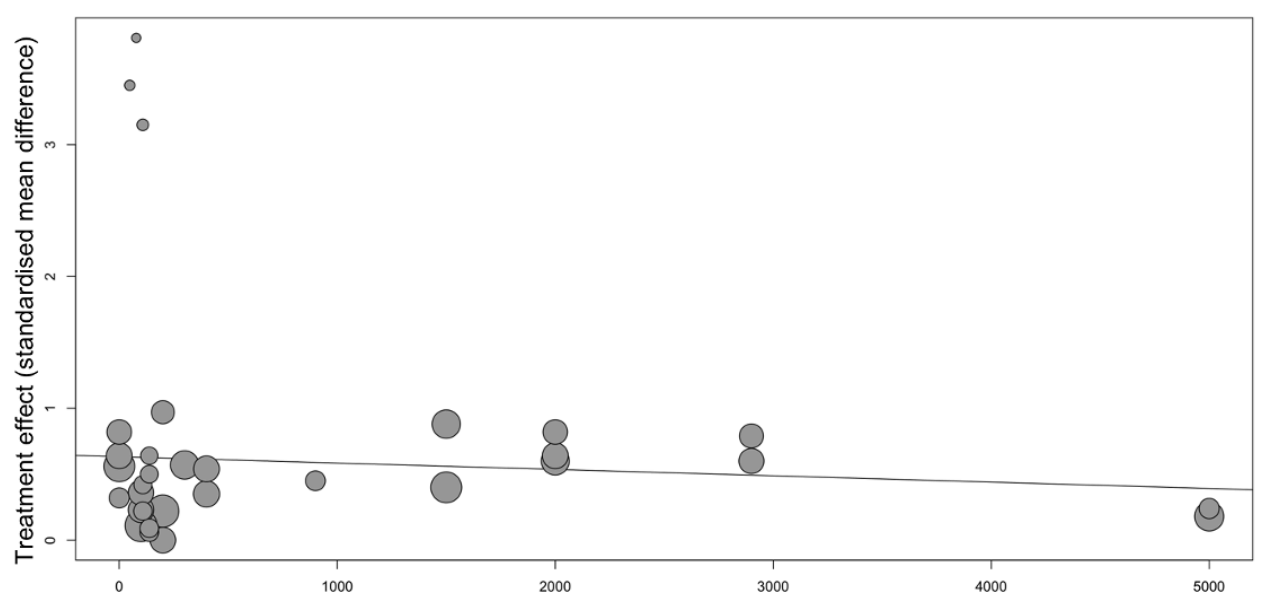

B

Covariate TMS after stimulus onset

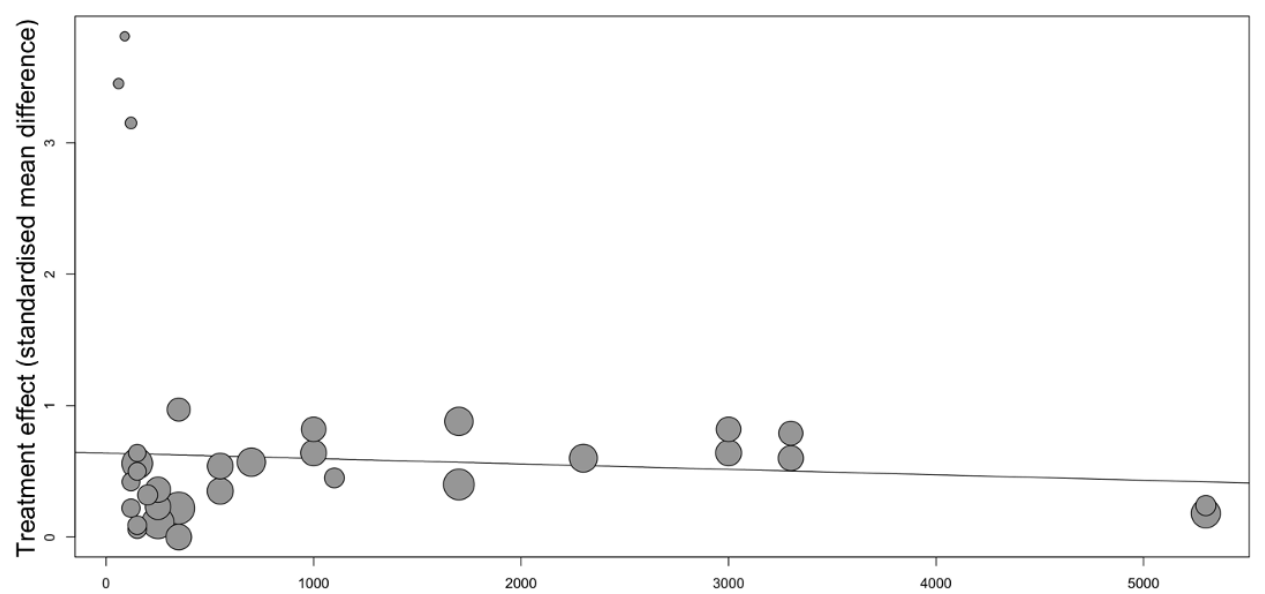

C

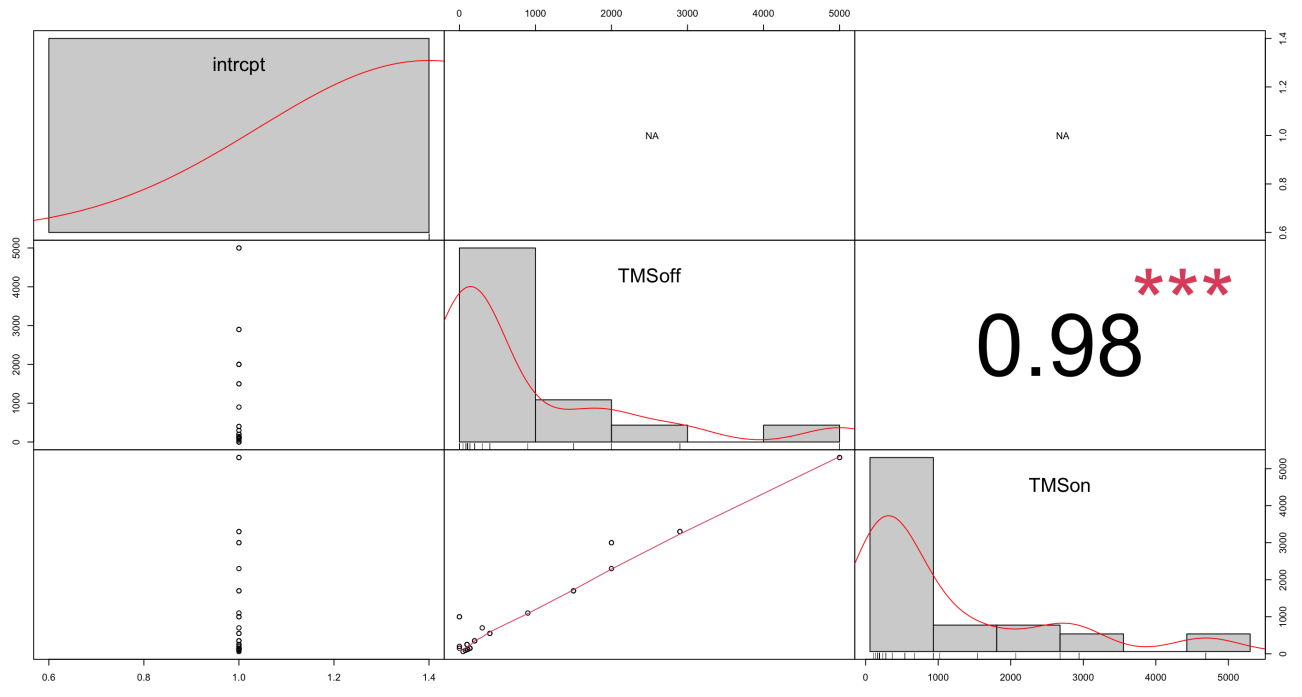

Fig 5. The bubble plot of meta-regressions of behavioral outcomes when inducing SVC TMS during a VSTM task, with the different TMS timing points after (A) stimulus offset and (b) stimulus onset used as a predictor variable. The TMS timings fail to predict the studies' effect sizes, as also confirmed by the (C) correlation analysis between the two metaregressions, indicating that the TMS effects are likely similar independent of the time during the VSTM task that stimulation is induced. SVC; sensory visual cortex, TMS; transcranial magnetic stimulation, VSTM; visual short-term memory. 
Table 3: Experiments included in the meta-analysis of TMS on SVC during the maintenance phase of a VSTM task.

\begin{tabular}{|c|c|c|c|c|c|c|c|c|}
\hline & STUDY & $\begin{array}{c}\text { EFFECT } \\
\text { SIZE }\end{array}$ & $\begin{array}{l}\text { STANDARD } \\
\text { ERROR }\end{array}$ & $\begin{array}{c}\text { Z- } \\
\text { VALUE }\end{array}$ & $\begin{array}{c}\text { P- } \\
\text { VALUE }\end{array}$ & $\mathbf{N}$ & $\begin{array}{l}\text { BEHAVIORAL } \\
\text { MEASURE } \\
\text { (condition) }\end{array}$ & $\begin{array}{l}\text { CONTROL } \\
\text { CONDITION }\end{array}$ \\
\hline 1 & $\begin{array}{l}\text { Cattaneo et al } \\
(2009) \mathrm{a}\end{array}$ & 0.82 & 0.32 & 2.55 & 0.01 & 14 & Accuracy & No TMS \\
\hline 2 & $\begin{array}{l}\text { Cattaneo et al } \\
(2009) \mathrm{b}\end{array}$ & 0.64 & 0.30 & 2.11 & 0.04 & 14 & Accuracy & Sham TMS \\
\hline 3 & $\begin{array}{l}\text { van de Ven et } \\
\text { al (2012)a }\end{array}$ & 0.35 & 0.30 & 1.17 & 0.24 & 12 & $\begin{array}{c}\text { Detection Sensitivity } \\
A^{\prime}(\mathrm{LL})\end{array}$ & Ipsilateral SVC \\
\hline 4 & $\begin{array}{l}\text { van de Ven et } \\
\text { al (2012)b }\end{array}$ & 0.54 & 0.30 & 1.78 & 0.08 & 12 & $\begin{array}{c}\text { Detection Sensitivity } \\
A^{\prime}(\mathrm{HL})\end{array}$ & Ipsilateral SVC \\
\hline 5 & $\begin{array}{l}\text { Saad et al } \\
(2013) \mathrm{a}\end{array}$ & 0.60 & 0.28 & 2.18 & 0.03 & 16 & $\begin{array}{l}\text { Accuracy (Exp2 } \\
2000 \mathrm{~ms})\end{array}$ & Sham TMS \\
\hline 6 & $\begin{array}{l}\text { Saad et al } \\
(2013) \mathrm{b}\end{array}$ & 0.18 & 0.26 & 0.69 & 0.49 & 16 & $\begin{array}{l}\text { Accuracy (Exp2 } \\
5000 \mathrm{~ms})\end{array}$ & Sham TMS \\
\hline 7 & $\begin{array}{l}\text { Saad et al } \\
(2013) c\end{array}$ & 0.24 & 0.39 & 0.61 & 0.54 & 8 & $\begin{array}{l}\text { Accuracy (Exp3 } \\
5000 \mathrm{~ms})\end{array}$ & Sham TMS \\
\hline 8 & $\begin{array}{l}\text { Zokaei et al } \\
(2014) \mathrm{a}\end{array}$ & 0.79 & 0.33 & 2.40 & 0.02 & 13 & $\begin{array}{l}\text { Precision (Exp } 1 \\
\text { congruent) }\end{array}$ & Sham TMS \\
\hline 9 & $\begin{array}{l}\text { Zokaei et al } \\
(2014) \mathrm{b}\end{array}$ & 0.6 & 0.31 & 1.91 & 0.06 & 13 & $\begin{array}{l}\text { Precision (Exp } 1 \\
\text { incongruent) }\end{array}$ & Sham TMS \\
\hline 10 & $\begin{array}{l}\text { Zokaei et al } \\
(2014) \mathrm{c}\end{array}$ & 0.57 & 0.27 & 2.11 & 0.04 & 17 & $\begin{array}{l}\text { Precision (Exp } 2 \\
\text { Item 1) }\end{array}$ & Sham TMS \\
\hline 11 & $\begin{array}{l}\text { Rademaker et } \\
\text { al (2017) }\end{array}$ & 0.45 & 0.40 & 1.13 & 0.26 & 8 & Absolute Error & Sham TMS \\
\hline 12 & $\begin{array}{l}\text { van } \\
\text { Lamsweerde et } \\
\text { al (2017)a }\end{array}$ & 0.22 & 0.22 & 0.98 & 0.33 & 21 & Guess Rate (200ms) & Ipsilateral SVC \\
\hline 13 & $\begin{array}{l}\text { Jia et al. } \\
\text { (2021)a }\end{array}$ & 0.4 & 0.24 & 1.13 & 0.26 & 20 & $\begin{array}{l}\text { Accuracy (pre- } \\
\text { training) }\end{array}$ & Sham TMS \\
\hline 14 & $\begin{array}{l}\text { Jia et al. } \\
(2021) b\end{array}$ & 0.88 & 0.27 & 3.25 & $<0.01$ & 20 & $\begin{array}{l}\text { Accuracy (post- } \\
\text { training) }\end{array}$ & Sham TMS \\
\hline
\end{tabular}

Tab 3. All effect sizes, standard errors, $95 \%$ confidence intervals, $Z$-values, $p$-values and weight calculated from the included studies that induced TMS during the maintenance phase of a VSTM task. HL; high load condition, LL; low load condition SVC; sensory visual cortex, TMS; transcranial magnetic stimulation, VSTM; visual short-term memory. 
Figure 6: Exploratory meta-analysis on the direction of SVC TMS on VSTM performance.

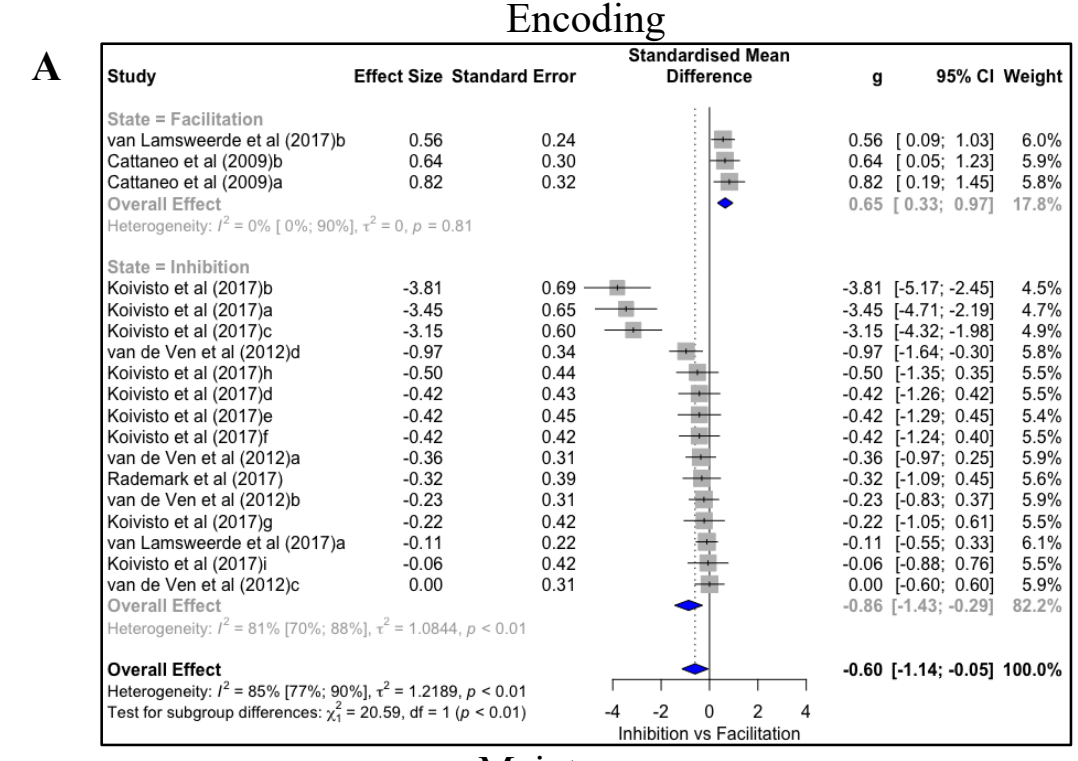

Maintenance

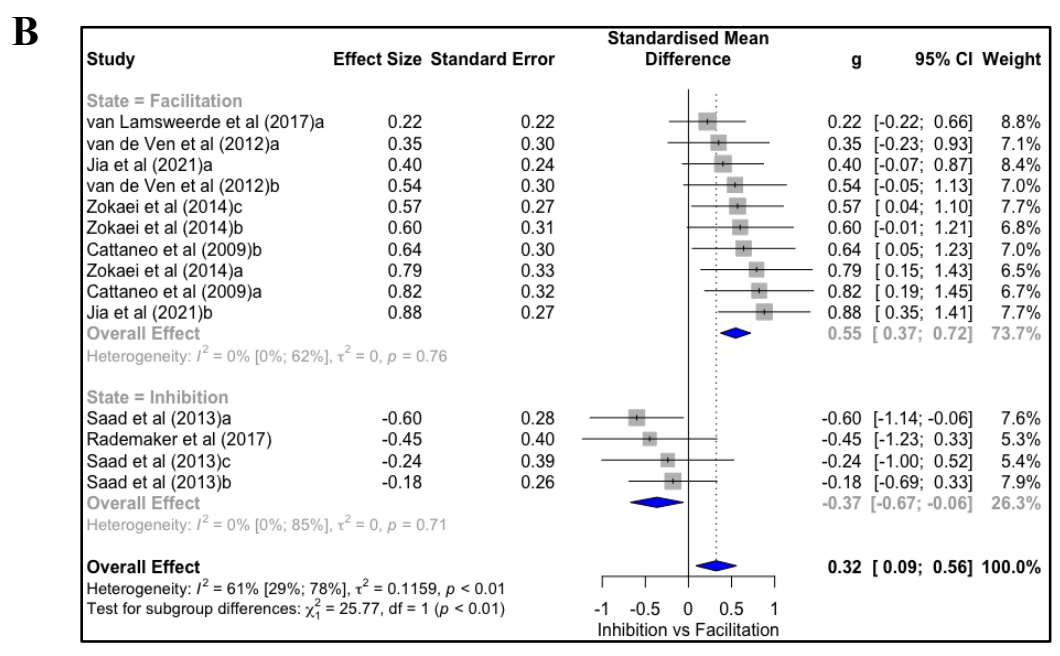

C

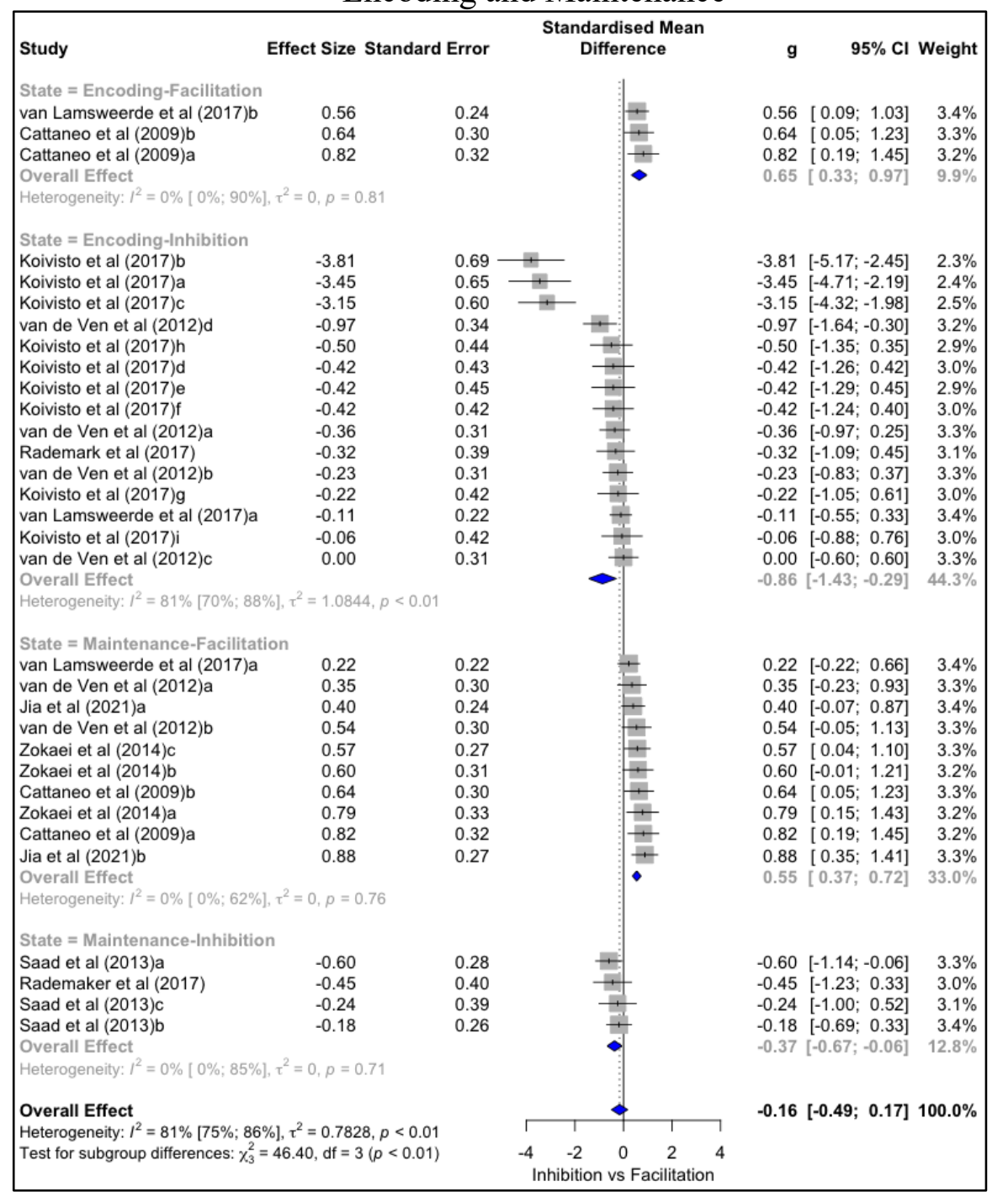


Fig 6. Exploring the direction of effects in (A) encoding, (B) maintenance, and (C) all effect sizes. For illustration purposes, effect sizes were categorized as either a facilitation or an inhibition effect. Results are inconclusive, with no evidence of neither facilitation nor inhibition effects of SVC TMS in VSTM behavioral outcomes.

Note: The signs of effect sizes from studies measuring guess rates (van Lamsweerde et al., 2017), proportion of guesses (Koivisto et al., 2017), and absolute errors (Rademaker et al., 2017) were reversed, in order to indicate the same direction of effect as those measuring percent correct and detection sensitivity.

SVC; sensory visual cortex, TMS; transcranial magnetic stimulation, VSTM; visual short-term memory. 